University of Nebraska - Lincoln

DigitalCommons@University of Nebraska - Lincoln

6-7-2007

\title{
Description of harmonic generation in terms of the complex quasienergy. I. General formulation
}

\author{
M.V. Frolov \\ Voronezh State University, Voronezh 394006, Russia
}

A.V. Flegel

Voronezh State University, Voronezh 394006, Russia

N. L. Manakov

Voronezh State University, manakov@phys.vsu.ru

Anthony F. Starace

University of Nebraska-Lincoln, astarace1@unl.edu

Follow this and additional works at: https://digitalcommons.unl.edu/physicsstarace

Part of the Physics Commons

Frolov, M.V.; Flegel, A.V.; Manakov, N. L.; and Starace, Anthony F., "Description of harmonic generation in terms of the complex quasienergy. I. General formulation" (2007). Anthony F. Starace Publications. 104. https://digitalcommons.unl.edu/physicsstarace/104

This Article is brought to you for free and open access by the Research Papers in Physics and Astronomy at DigitalCommons@University of Nebraska - Lincoln. It has been accepted for inclusion in Anthony F. Starace Publications by an authorized administrator of DigitalCommons@University of Nebraska - Lincoln. 


\title{
Description of harmonic generation in terms of the complex quasienergy. I. General formulation
}

\author{
M. V. Frolov, A. V. Flegel, and N. L. Manakov \\ Department of Physics, Voronezh State University, Voronezh 394006, Russia \\ Anthony F. Starace \\ Department of Physics and Astronomy, University of Nebraska, Lincoln, Nebraska 68588-0111, USA
}

(Received 16 January 2007; published 7 June 2007)

\begin{abstract}
Using the Hellmann-Feynman theorem for quasistationary quasienergy states of a quantum system in an intense laser field, we present the high-order harmonic generation (HHG) amplitude in terms of the complex quasienergy of a bound electron, thereby avoiding the necessity for an explicit form for the electron wave function in HHG calculations. This formulation for the HHG amplitude confirms use of the dual dipole moment (instead of the dipole moment expectation value) in wave-function-based HHG calculations in order to properly account for both the ionization and the Stark shift of the initial bound state in a strong laser field.

DOI: $10.1103 /$ PhysRevA.75.063407

PACS number(s): $32.80 . \mathrm{Wr}, 42.50 . \mathrm{Hz}, 42.65 . \mathrm{Ky}$
\end{abstract}

\section{INTRODUCTION}

The first evidence of the highly nonperturbative interaction of an intense laser field with matter in the spectra of high-order harmonics was obtained in the late 1980s in experiments involving rare gas atoms $[1,2]$. These experiments showed that although the first few harmonics drop rapidly in intensity, a plateau is reached along which harmonics have roughly comparable intensities. One of the experiments [2] demonstrated also that beyond some harmonic energy on the plateau (the "cutoff" energy), subsequent harmonics once again drop rapidly in intensity with increasing harmonic order. The existence of a plateau of harmonic intensities stimulated efforts to extend the cutoff energy into the so-called "water window" region of the x-ray spectrum (since coherent $\mathrm{x}$-ray sources in this region would enable researchers to image living biological structures). Within a decade, success in obtaining x-ray harmonics in the "water window" region was achieved $[3,4]$. High-harmonic sources with photon energies of about $40 \mathrm{eV}$ and intensities of about $10^{14} \mathrm{~W} / \mathrm{cm}^{2}$ are currently available for laboratory experiments (see, e.g., Ref. [5]). Investigations of high-order harmonic generation (HHG) have taken on additional importance at present owing to applications in attosecond science [6]. Specifically, plateau harmonics have been used as sources of both attosecond pulse trains [7] and of single attosecond pulses [8,9]. Such pulses (having durations in the attosecond regime) have been employed to obtain time-resolved measurements of electronic processes in atoms [8]. HHG thus appears to be a fundamental component of attosecond science.

Owing to the intrinsic interest and the important applications of the plateau structures in HHG spectra, theorists have sought to obtain a fundamental understanding of these highly nonperturbative HHG features and also to discover means to control them. As there have appeared a number of recent reviews that discuss the theory of HHG (see, e.g., Refs. [10-14]), we confine ourselves here to only a few remarks relevant to the content of this paper. A key step in the development of current theoretical understanding was the initial observation of an empirical $\left|E_{0}\right|+3 u_{p}$ law for the energy position of the HHG plateau cutoff [15] (where $\left|E_{0}\right|$ is the atomic binding energy and $u_{p}$ is the ponderomotive potential of an electron in a laser field). This law was interpreted in terms of the so-called "simple man model" (in which an atomic electron tunnels out of an atom owing to the lowering of the potential barrier by the laser field, is driven away from and then back to the atomic core by the laser field, whereupon it may release its accumulated kinetic energy in the form of harmonic photons) [16-18]. The success of this single-active electron picture in predicting a key feature of the HHG plateau led to single-active electron quantum formulations that are consistent with this picture (see, e.g., Refs. [19-22], and references therein). These theories are based on the so-called "strong field approximation" in which, after tunneling from the atom, the active electron is described by a Volkov wave function, i.e., the atomic potential and all of its bound states (except for the ground state) are ignored.

Despite these clear successes, however, evidence of the need for an improved theoretical formulation of HHG has appeared. First, HHG rates have been found to be very sensitive to the bound (initial) state wave function employed, especially for low harmonic orders and nonzero angular momentum of the bound electron [23]. Second, for intense laser fields, the shift and ionization broadening of the initial state energy by a strong laser field must be taken into account, which is generally not done by most current theoretical methods. Moreover, a number of analytical and semianalytical methods that originate from formulations claimed to be "exact" or "ab initio" yield different final results after making apparently the same chain of approximations. There thus appears to be a need for a rigorous quantum formulation for the HHG amplitude and for the development of semianalytical quantum models that are consistent with this formulation and applicable over a wide range of laser parameters (in particular, frequency and intensity). These issues motivate the present paper as well as the following paper [24].

The HHG process for a single bound electron driven by an intense monochromatic laser field is formulated in this paper in terms of the system's quasienergy, thereby avoiding the necessity for an explicit form of the electron wave function to calculate HHG rates. This formulation makes use of the Hellmann-Feynman theorem $[25,26]$ for the quasistationary quasienergy states (QQES) (or Floquet states) of the system. Because of its focus on the complex quasienergy of the 
system, our formulation automatically includes both the Stark shift and the ionization width of the initial bound state in a strong laser field. Moreover, our wave-functionindependent formulation yields a result for the HHG amplitude that is equivalent to that obtained using properly normalized QQES wave functions, i.e., involving dual QQES wave functions to obtain so-called dual dipole moments. Using our exact and approximate results for this dipole moment, we analyze the connection between various common semianalytical HHG theories and exhibit their differences for the case of a zero-range potential (ZRP) model (for a bound electron in an $s$ state), which is an exactly solvable HHG problem. In addition, we discuss the definition of $\mathrm{HHG}$ rates for atomic systems having a nonzero, randomly oriented total angular momentum.

Finally, in this paper we focus on the single atom or ion response to a driving (monochromatic) laser field. We analyze neither propagation effects on the harmonics nor pulse shape effects. However, as long as the driving laser pulse is long (i.e., contains many laser cycles) and the paraxial approximation is valid (i.e., the laser focal dimension is much greater than the laser wavelength), then the calculation of the single atom response can be carried out separately from the calculation of the propagation of the harmonics generated in the atomic medium [11]. Also, only in this (monochromatic) approximation may we introduce the correct definition of HHG rates, which provide a measure of those atomic parameters that depend only on laser intensity and carrier frequency.

This paper is organized as follows. In Sec. II we discuss in some detail current theoretical approaches to describing $\mathrm{HHG}$ in terms of the field-induced dipole moments of an atomic system. In Sec. III we formulate an expression for HHG amplitudes in terms of a system's complex quasienergy. In Sec. IV we connect the formulation in terms of complex quasienergy (or, equivalently, in terms of the dual dipole moment) to commonly used alternative approaches for HHG rates. In Sec. V we discuss the definition of $\mathrm{HHG}$ rates for the case of a bound electron with nonzero angular momentum and generalize the results of Sec. III to this case. In Sec. VI we summarize the key results of this paper and present some conclusions. Derivations of analytic results for induced dipole moments and corresponding nonlinear susceptibilities for a ZRP model, used in Sec. IV, are given in the Appendix.

\section{ON THE QUANTUM DEFINITION OF THE SINGLE-ATOM HHG AMPLITUDE IN TERMS OF AN INDUCED DIPOLE MOMENT}

For a single atom, the quantum definition of the HHG amplitude in terms of a field-induced dipole moment is attractive since this description is closely related to the classical picture of harmonic generation by an anharmonic oscillator [e.g., by a classical electron bound in a static potential, $U(x)$ ] subjected to a strong monochromatic perturbation. However, the quantum generalization of this classical picture is complicated by the fact that harmonic generation takes place simultaneously with the competing process of multiphoton ionization, which must thus be taken into account.
For this reason, the use of such sophisticated quantum approaches as quantum electrodynamics or formal $S$-matrix methods are not the best choices for obtaining an accurate quantum definition of the single-atom HHG amplitude. The major concern is that while these methods are well-justified mathematically for collision problems, which involve scattering (i.e., continuum) states, in the case of single-atom HHG both the initial and final states are bound and thus their modification (or "dressing") by a strong laser field, including ionization effects, must be taken into account (see Ref. [27] for additional discussion). In this paper, we employ the QQES (or Floquet) method to define accurately the quantum amplitude for HHG by an atomic system in an initial (bound) state of energy $E_{0}$ with proper account for the field-induced shift of the energy $E_{0}$ and its broadening (the ionization width) due to the ionization processes that accompany harmonic generation. The principal features of this definition may be most clearly presented for the simplest, single-active electron model of the laser-atom interaction, i.e., the electricdipole interaction,

$$
V(\mathbf{r}, t)=-\frac{1}{2} e F\left[(\mathbf{e} \cdot \mathbf{r}) e^{-i \omega t}+\left(\mathbf{e}^{*} \cdot \mathbf{r}\right) e^{i \omega t}\right],
$$

which describes the interaction of a long monochromatic laser pulse (having an electric vector $\mathbf{F}(t)$ $=F \operatorname{Re}[\mathbf{e} \exp (-i \omega t)]\left(\mathbf{e} \cdot \mathbf{e}^{*}=1\right)$ and an intensity $I=\left(c F^{2}\right) /$ $(8 \pi)$ ) with an electron that is subject also to a static potential, $U(\mathbf{r})$, that supports both bound $\left[\psi_{n}(\mathbf{r})\right]$ and continuum $\left[\psi_{E}(\mathbf{r})\right]$ stationary states. An initial bound state, $\psi_{0}(\mathbf{r}, t)$ $=\psi_{0}(\mathbf{r}) e^{-i E_{0} t / \hbar}$, of an electron in the potential $U(\mathbf{r})$ evolves as the perturbation $V(\mathbf{r}, t)$ is turned on adiabatically according to the time-dependent Schrödinger equation (TDSE),

$$
\left(-\frac{\hbar^{2}}{2 m} \nabla^{2}+U(\mathbf{r})+V(\mathbf{r}, t)-i \hbar \frac{\partial}{\partial t}\right) \Psi(\mathbf{r}, t)=0 .
$$

In order to organize our discussion in this longer section, we divide it into three parts. We first discuss certain inconsistencies in a common perturbative approach for nonlinear susceptibilities. We then discuss briefly the quasienergy approach for both perturbative and nonperturbative regimes of the laser-atom interaction. Finally we introduce the concept of the so-called "dual" dipole moment for the correct definition of the HHG amplitude in the strong field regime.

\section{A. Inconsistency of the standard perturbative approach for nonlinear susceptibilities at above-threshold frequencies}

Consider first the case of a not too strong laser field so that perturbation theory (PT) may be used to treat the interaction $V(\mathbf{r}, t)$ in Eq. (2), including possibly higher-order PT corrections to the lowest-order PT result. This formulation is typical for traditional ("perturbative") nonlinear optics, in which case the generation of harmonics by an ensemble of atoms subjected to a long monochromatic laser pulse of frequency $\omega$ is described by the atomic response at the harmonic frequency $\Omega=N \omega$, which is given by the Fourier component $\mathbf{d}_{\Omega}$ of the light-induced dipole moment $\mathbf{d}(t)$ of the atom at the harmonic frequency $\Omega$. This Fourier component is obtained from the perturbation expansion (in $V$ ) of $\mathbf{d}(t)$, 
which is commonly defined as the expectation value of the dipole moment operator, $\mathbf{d}=e \mathbf{r}$,

$$
\mathbf{d}(t)=\langle\Psi(t)|\mathbf{d}| \Psi(t)\rangle=\frac{1}{2} \sum_{n=0}^{\infty}\left(\mathbf{d}_{n \omega} e^{-i n \omega t}+\mathbf{d}_{-n \omega} e^{i n \omega t}\right),
$$

where $\Psi(t) \equiv \Psi(\mathbf{r}, t)$ is the PT solution of the TDSE (2) corresponding to the initial state $\psi_{0}(\mathbf{r})$ and where only odd values of $n$ contribute for systems having inversion symmetry. Defining the PT parameter as the ratio of the laser amplitude $F$ to the characteristic strength $F_{0}$ of the internal atomic electric field (e.g., $F_{0}=e / a_{0}^{2} \approx 5.14 \times 10^{9} \mathrm{~V} / \mathrm{cm}$ for the hydrogen atom), the vector $\mathbf{d}_{N \omega}$ may be expressed as a series expansion in terms of nonlinear susceptibility tensors, $\chi_{N+2 k}(N \omega)(k$ $=0,1,2, \ldots)$, which depend on the frequency of the pump (laser) field. In lowest order, $\mathbf{d}_{N \omega}$ is proportional to $\chi_{N}(N \omega) F^{N}$; the next-order term in $F$ is proportional to $\chi_{N+2}(N \omega) F^{N+2}$, and so on. Note that in order to keep our notation as simple as possible, we omit here the tensor indices of $\chi_{N+2 k}(N \omega)$, which is a Cartesian tensor of rank $N$ $+2 k+1$ (see, e.g., Ref. [28]).

We have started with the PT regime of laser-atom interactions in order to show that even in this regime there is an inconsistency in the standard definition (3) for $\mathbf{d}(t)$ in higher orders of PT. The problem arises when taking into account high-order PT corrections to $\mathbf{d}_{N \omega}$ for the case of $N \geqslant n_{0}$, where $n_{0}$ is the threshold number of photons for ionization from the state $\psi_{0}(\mathbf{r})$, i.e., $n_{0}$ is the smallest integer for which $n_{0} \hbar \omega>\left|E_{0}\right|$. In this case the high-order PT matrix elements for the susceptibilities $\chi_{N+2 k}(N \omega)$, defined by the standard (Rayleigh-Schrödinger) PT expansion of the matrix element $\langle\Psi(t)|\mathbf{d}| \Psi(t)\rangle$ in Eq. (3), become divergent. This fact was demonstrated in Ref. [29] in a calculation of the dynamic hyperpolarizability, $\gamma(\omega) \equiv \chi_{3}(\omega)$, which determines the lowest-order (i.e., linear in intensity $I$ ) correction to the ordinary dynamic polarizability of an atom in the state $\psi_{0}(\mathbf{r})$, $\alpha(\omega) \equiv \chi_{1}(\omega)$, for the case of above-threshold frequencies, $\hbar \omega>\left|E_{0}\right|$. The origin of the divergences is as follows: the PT expansion of $|\Psi(\mathbf{r}, t)\rangle$ in Eq. (3) contains outgoing wave Green functions, $G_{E+i 0}\left(\mathbf{r}, \mathbf{r}^{\prime}\right)$, of the electron in the potential $U(\mathbf{r})$, which are complex when $E=\left(E_{0}+p \hbar \omega\right)>0$ (i.e., for $\left.p \geqslant n_{0}\right)$ and whose spectral decompositions,

$$
G_{E+i 0}\left(\mathbf{r}, \mathbf{r}^{\prime}\right)=\sum_{n} \frac{\psi_{n}(\mathbf{r}) \psi_{n}^{*}\left(\mathbf{r}^{\prime}\right)}{E-E_{n}}+\int_{0}^{\infty} d E^{\prime} \frac{\psi_{E^{\prime}}(\mathbf{r}) \psi_{E^{\prime}}^{*}\left(\mathbf{r}^{\prime}\right)}{E-E^{\prime}+i 0},
$$

involve (regularizable) singular terms, $\sim 1 /\left(E-E^{\prime}+i 0\right)$, in integrals over the energy $E^{\prime}$ of intermediate continuum states. Since $\mathbf{d}(t)$ in Eq. (3) also contains the bra-vector, $\langle\Psi(\mathbf{r}, t)|$, the PT expansion of this state vector involves complex-conjugated (ingoing wave) Green functions, $G_{E-i 0}\left(\mathbf{r}, \mathbf{r}^{\prime}\right)\left(G_{E-i 0}=G_{E+i 0}^{*}\right)$, having the singular terms $\sim 1 /\left(E-E^{\prime \prime}-i 0\right)$ in integrals over $E^{\prime \prime}$. Thus the resulting expressions for high-order susceptibilities at above-threshold frequencies involve double integrals over $E^{\prime}$ and $E^{\prime \prime}$ which contain nonregularizable (and thus nonintegrable) singularities $\sim 1 /\left[\left(E-E^{\prime}+i 0\right)\left(E-E^{\prime}-i 0\right)\right]$ on the line $E^{\prime}=E^{\prime \prime}$. [For example, for the case of linearly polarized $\mathbf{F}(t)$, the hyperpolarizability $\gamma(\omega)$ includes the following two fourth-order PT matrix elements,

$$
\begin{gathered}
\left\langle\psi_{0}\left(\mathbf{r}_{4}\right)\right| z_{4} G_{E_{0}+\hbar \omega-i 0}\left(\mathbf{r}_{4}, \mathbf{r}_{3}\right) z_{3} G_{E_{0}+2 \hbar \omega+i 0}\left(\mathbf{r}_{3}, \mathbf{r}_{2}\right) \\
\times z_{2} G_{E_{0}+\hbar \omega+i 0}\left(\mathbf{r}_{2}, \mathbf{r}_{1}\right) z_{1}\left|\psi_{0}\left(\mathbf{r}_{1}\right)\right\rangle, \\
\left\langle\psi_{0}\left(\mathbf{r}_{4}\right)\right| z_{4} G_{E_{0}+\hbar \omega-i 0}\left(\mathbf{r}_{4}, \mathbf{r}_{3}\right) z_{3} G_{E_{0}}^{(0)}\left(\mathbf{r}_{3}, \mathbf{r}_{2}\right) \\
\times z_{2} G_{E_{0}+\hbar \omega+i 0}\left(\mathbf{r}_{2}, \mathbf{r}_{1}\right) z_{1}\left|\psi_{0}\left(\mathbf{r}_{1}\right)\right\rangle,
\end{gathered}
$$

which diverge at $\left(E_{0}+\hbar \omega\right)>0$. The superscript $(0)$ in $G_{E_{0}}^{(0)}$ indicates that the term with $n=0$ in the sum over $n$ is omitted in the spectral expansion (4) for this function.] Physically, the occurrence of these divergences means that the definition (3) is appropriate only for a stable system (e.g., one without a continuous spectrum), in which case the "field-dressed" wave function $\Psi(\mathbf{r}, t)$ is normalizable to unity in the usual way, $\langle\Psi(t) \mid \Psi(t)\rangle=1$, in each PT order. For a decaying system [e.g., one described by the wave function $\Psi(\mathbf{r}, t)$ that takes into account open ionization channels], the expectation value of $\mathbf{d}$ diverges since $\Psi(\mathbf{r}, t)$ involves a continuum component (see Ref. [30] for further discussion). Therefore, the "classical" definition (3) of the dipole moment is not appropriate for a correct description of the atomic response to a monochromatic perturbation when taking into account the accompanying ionization effects even in the PT approach (except for the lowest nonvanishing PT order for the nonlinear susceptibilities, in which case the PT matrix elements do not involve Green functions $G_{E_{1}+i 0}$ and $G_{E_{2}-i 0}$ with the same energies, $E_{1}=E_{2}$ ).

\section{B. Quasienergy analysis of nonlinear susceptibilities}

For a proper quantum definition of the atomic response at the frequency $\Omega=N \omega$ for both perturbative (including highorder PT terms) and nonperturbative treatments of $V(\mathbf{r}, t)$, it is convenient to use the quasienergy (or Floquet) solution of the TDSE (2) since it explicitly takes into account the fieldinduced shift and width of the initial-bound-state energy, $E_{0}$ (see, e.g., Ref. [31]). This solution has the following form:

$$
\Psi_{\epsilon}(\mathbf{r}, t)=e^{-i \epsilon t / \hbar} \Phi_{\epsilon}(\mathbf{r}, t)=\sum_{s} \Phi_{s}(\mathbf{r}) e^{-(i / \hbar)(\epsilon+s \hbar \omega) t},
$$

where the QQES wave function, $\Phi_{\epsilon}(\mathbf{r}, t)$, corresponding to $\psi_{0}(\mathbf{r})$, is the solution of the non-Hermitian eigenvalue equation in a space of periodic functions $\left[\Phi_{\epsilon}(\mathbf{r}, t+T)\right.$ $\left.=\Phi_{\epsilon}(\mathbf{r}, t), T=2 \pi / \omega\right]$,

$$
\begin{aligned}
\hat{\mathcal{H}}(\mathbf{r}, t) \Phi_{\epsilon}(\mathbf{r}, t) & \equiv\left(-\frac{\hbar^{2}}{2 m} \nabla^{2}+U(\mathbf{r})+V(\mathbf{r}, t)-i \hbar \frac{\partial}{\partial t}\right) \Phi_{\epsilon}(\mathbf{r}, t) \\
& =\epsilon \Phi_{\epsilon}(\mathbf{r}, t),
\end{aligned}
$$

for the complex quasienergy $\epsilon=\operatorname{Re} \epsilon-i(\hbar / 2) \Gamma$, where $\Delta \epsilon$ $=\operatorname{Re} \epsilon-E_{0}$ and $\Gamma$ are the energy shift and the total decay rate of the state $\psi_{0}(\mathbf{r})$. The fact that $\epsilon$ is complex is ensured by the complex boundary condition for $\Phi_{\epsilon}(\mathbf{r}, t)$ at $r \rightarrow \infty$ : the square root in $\hbar k_{n}=\sqrt{2 m\left(n \hbar \omega+\epsilon-u_{p}\right)}$ [where $u_{p}$ is the pon- 
deromotive energy, $\left.\left(e^{2} F^{2}\right) /\left(4 m \omega^{2}\right)\right]$ is chosen so that in the open ionization channels $\left(\operatorname{Re} k_{n}^{2}>0\right)$ the Fourier-components of $\Phi_{\epsilon}(\mathbf{r}, t), \Phi_{s}(\mathbf{r})$, describe outgoing spherical waves, i.e., $\operatorname{Re} k_{n}>0$, while in the closed channels $\left(\operatorname{Re} k_{n}^{2}<0\right)$ they describe exponentially damped waves, i.e., $\operatorname{Im} k_{n}>0$.

The integral form of the QQES equation (7) is useful in many instances (cf. Ref. [32]). In the strong field (nonperturbative) regime, it may be written in terms of the (retarded) Green's function, $G^{(V)}\left(\mathbf{r}, t ; \mathbf{r}^{\prime}, t^{\prime}\right)$, of a free electron in the laser field $\mathbf{F}(t)$ [cf. Appendix A for its explicit form; note that $\left.G^{(V)}\left(\mathbf{r}, t+T ; \mathbf{r}^{\prime}, t+T-\tau\right)=G^{(V)}\left(\mathbf{r}, t ; \mathbf{r}^{\prime}, t-\tau\right)\right]$,

$$
\begin{aligned}
\Phi_{\epsilon}(\mathbf{r}, t)= & \int d \mathbf{r}^{\prime} \int_{-\infty}^{t} d t^{\prime} e^{i \epsilon\left(t-t^{\prime}\right) / \hbar} G^{(V)}\left(\mathbf{r}, t ; \mathbf{r}^{\prime}, t^{\prime}\right) \\
& \times U\left(\mathbf{r}^{\prime}\right) \Phi_{\epsilon}\left(\mathbf{r}^{\prime}, t^{\prime}\right) \\
= & \int d \mathbf{r}^{\prime} \int_{0}^{\infty} d \tau e^{i \epsilon \tau / \hbar} G^{(V)}\left(\mathbf{r}, t ; \mathbf{r}^{\prime}, t-\tau\right) U\left(\mathbf{r}^{\prime}\right) \\
& \times \Phi_{\epsilon}\left(\mathbf{r}^{\prime}, t-\tau\right),
\end{aligned}
$$

which is especially useful for finite-range potentials, $U(\mathbf{r})$, and permits an exact solution of the QQES problem for the widely used zero-range potential (ZRP) model $[33,34]$. In the perturbative regime, the PT expansions for $\epsilon$ and $\Phi_{\epsilon}(\mathbf{r}, t)$ may be obtained by converting the differential equation (7) into an inhomogeneous integral equation involving the "quasienergy Green function" of an electron in the potential $U(\mathbf{r}), \mathcal{G}_{\epsilon}\left(\mathbf{r}, t ; \mathbf{r}^{\prime}, t^{\prime}\right)=\Sigma_{k} \exp \left[i k \omega\left(t-t^{\prime}\right)\right] G_{\epsilon-k \hbar \omega}^{(0)}\left(\mathbf{r}, \mathbf{r}^{\prime}\right)$ [where the superscript (0) in $G_{\epsilon-k \hbar \omega}^{(0)}$ means that, at $k=0$, the term with $n=0$ in the sum over $n$ is omitted in Eq. (4) for this function],

$$
\begin{aligned}
\Phi_{\epsilon}(\mathbf{r}, t)= & \psi_{0}(\mathbf{r})+\frac{1}{T} \int_{0}^{T} d t^{\prime} \int d \mathbf{r}^{\prime} \\
& \times \mathcal{G}_{\epsilon}\left(\mathbf{r}, t ; \mathbf{r}^{\prime}, t^{\prime}\right) V\left(\mathbf{r}^{\prime}, t^{\prime}\right) \Phi_{\epsilon}\left(\mathbf{r}^{\prime}, t^{\prime}\right) .
\end{aligned}
$$

Together with the "Brillouin-Wigner relation" for $\epsilon$ and $E_{0}$,

$$
\epsilon=E_{0}+\frac{1}{T} \int_{0}^{T}\left\langle\psi_{0}(\mathbf{r})\left|V\left(\mathbf{r}, t^{\prime}\right)\right| \Phi_{\epsilon}\left(\mathbf{r}, t^{\prime}\right)\right\rangle d t^{\prime}
$$

(which is similar to that for the exact and unperturbed energies in time-independent problems; see, e.g., Ref. [35]), Eq. (9) represents an eigenvalue problem for $\epsilon$ and $\Phi_{\epsilon}(\mathbf{r}, t)$ in a form that is convenient for a PT treatment. In this formulation, the perturbative expansion for $\epsilon$ in a power series in $F^{2}$,

$$
\epsilon=E_{0}+\sum_{n=1}^{\infty} \beta_{2 n}(\omega) F^{2 n},
$$

can be obtained from Eq. (10) by substituting the iterative solution for $\Phi_{\epsilon}\left(\mathbf{r}, t^{\prime}\right)$ that follows from Eq. (9) (see Refs. $[31,36]$ for further details).

The high-order PT corrections to $E_{0}, \epsilon_{2 n}=\beta_{2 n}(\omega) F^{2 n}$, become complex for $n \geqslant n_{0}$ and their imaginary parts determine the PT expansion of the decay rate $\Gamma$. We emphasize that, in contrast to $\mathbf{d}(t)$ in Eq. (3), the matrix element on the righthand side of Eq. (10) involves only the ket-vector $|\Phi(\mathbf{r}, t)\rangle$ [whose PT expansion involves only outgoing wave Green functions $G_{E+i 0}\left(\mathbf{r}, \mathbf{r}^{\prime}\right)$, so that the high-order PT matrix elements for $\epsilon_{2 n}$ do not involve the nonintegrable singularities discussed above and hence all coefficients $\beta_{2 n}(\omega)$ are finite [36]. Moreover, the explicit form of the coefficients $\beta_{2 n}(\omega)$ in terms of high-order PT matrix elements may be formally represented in terms of susceptibilities $\chi_{2 n-1}(\omega)$ [which define the PT expansion of the Fourier component $\mathbf{d}_{\omega}$ of the dipole moment $\mathbf{d}(t)$ in Eq. (3)], provided, however, that one takes in the matrix elements for $\chi_{2 n-1}(\omega)$ all Green functions that arise from the PT expansion of $\langle\Psi(t)|$ in Eq. (3) without complex conjugation, i.e., with the same sign of the infinitesimal, $i 0$, as in $G_{E+i 0}$ in Eq. (4). (For $n<n_{0}$, both $\beta_{2 n}(\omega)$ and $\chi_{2 n-1}(\omega)$ are real since the energy parameters $E=E_{0}$ $+p \hbar \omega(p \leqslant n)$ in all Green functions are negative and thus the infinitesimal $\pm i 0$ in Eq. (4) has no consequence. For this case, the exact relation between $\beta_{2 n}(\omega)$ and $\chi_{2 n-1}(\omega)$ follows from the Hellmann-Feynman theorem in the (real-valued) quasienergy approach for potentials $U(\mathbf{r})$ supporting only bound stationary states [see Ref. [37] and, also, the discussion of Eqs. (21) and (23) at the end of Sec. III below].) This recipe indicates how one can avoid the divergences [i.e., the appearance of ingoing wave Green functions $G_{E-i 0}\left(=G_{E+i 0}^{*}\right)$ with $E>0$ ] in calculations of high-order nonlinear susceptibilities using PT expansions of $\mathbf{d}(t)$ in Eq. (3). Namely, one should use in Eq. (3) in place of the bra-vector $\langle\Psi(\mathbf{r}, t)|$ $=\Psi^{*}(\mathbf{r}, t)$ the wave function $\Psi(\mathbf{r}, t)$ without complex conjugation but with the substitution $t \rightarrow-t$, i.e., $\langle\Psi(\mathbf{r}, t)| \rightarrow \Psi(\mathbf{r}$, $-t)$. This simple prescription is valid for a nondegenerate initial state $\psi_{0}(\mathbf{r})$ in a linearly polarized field $\mathbf{F}(t)$. However, if the polarization of $\mathbf{F}(t)$ is not linear, the substitution $\mathbf{e}$ $\rightarrow \mathbf{e}^{*}$ should also be made. In addition, if $\Psi(\mathbf{r}, t)$ depends on the signs of the magnetic quantum numbers $m$, then they should be changed to the opposite ones, $m \rightarrow-m$. Therefore, even in the PT regime, in order to obtain finite values of the high-order PT corrections to the nonlinear susceptibilities $\chi_{n}(n \omega)$ at energies corresponding to open multiphoton ionization channels (i.e., for $n>n_{0}$ ), the following substitution,

$$
\left\langle\Psi(\mathbf{r}, t)|\rightarrow \Psi(\mathbf{r},-t)|_{m \rightarrow-m, \mathbf{e} \rightarrow \mathbf{e}^{*}},\right.
$$

should be used in the definition (3) of the field-induced dipole moment of the nonstable (due to ionization) atomic system initially in the bound state $\psi_{0}(\mathbf{r})$.

In the PT regime, the correct definition of the HHG amplitude taking into account ionization effects and including high-order corrections, $\chi_{N+2 k}(N \omega)$, to $\chi_{N}(N \omega)$ up to some (finite) order $k$ may also be obtained unambiguously [i.e., without, in fact, employing the prescription (12)] as the transition amplitude between the same initial and final states, $\psi_{0}(\mathbf{r})$, for the process of absorption of $N$ laser photons followed by the emission of a spontaneous harmonic photon in the direction of the laser beam. In this way, both the lowestorder PT amplitude and high-order PT corrections to it (with each higher order describing virtual absorption and emission of an additional pair of laser photons) involve only outgoing wave Green functions $G_{E+i 0}$. With proper handling of the so-called secular and normalization terms that appear in high 
orders of PT for the case of a monochromatic perturbation (see, e.g., Refs. [31,38]), all high-order PT matrix elements are convergent at any frequency $\omega$. Furthermore, the results coincide with those obtained from Eq. (3) using the prescription (12). This agreement thus justifies this prescription for calculations of high-order nonlinear susceptibilities with proper account of ionization effects on the PT level in terms of the (modified) dipole moment.

\section{Nonperturbative regime of laser-atom interactions: The dual dipole moment}

Owing to its difficulties (i.e., its singularities) in the PT regime, Eq. (3) for the field-induced dipole moment may be expected to present problems also in the nonperturbative (strong field) regime. However, the prescription (12) proves to be useful for defining the HHG amplitude also in the nonperturbative regime, for which a PT expansion of the solution of equations (2) or (7) is not applicable. Indeed, it is necessary in this case to deal with the entire set of high-order corrections $\chi_{N+2 k}(N \omega)$ (i.e., for all $k$ ) to the lowest-order PT result $\chi_{N}(N \omega)$. In this regime the QQES solution (6) describes a quasistationary (decaying) state. Thus the usual (Hermitian) theory of quantum transitions cannot be used to justify the prescription (12), in part because the function $\Phi_{\epsilon}(\mathbf{r}, t)$ itself is formally divergent at $r \rightarrow \infty$ (owing to the asymptotically divergent waves in the ionization channels) and thus cannot be normalized to unity in the standard way. The proper normalization of $\Phi_{\epsilon}(\mathbf{r}, t)$ is achieved instead by introducing a "dual" function, $\widetilde{\Phi}_{\epsilon}(\mathbf{r}, t)$, such that

$$
\frac{1}{T} \int_{0}^{T} d t\left\langle\widetilde{\Phi}_{\epsilon}(t) \mid \Phi_{\epsilon}(t)\right\rangle=\frac{1}{T} \int_{0}^{T} d t \int d \mathbf{r}\left[\widetilde{\Phi}_{\epsilon}(\mathbf{r}, t)\right]^{*} \Phi_{\epsilon}(\mathbf{r}, t)=1 .
$$

The explicit form of $\widetilde{\Phi}_{\epsilon}(\mathbf{r}, t)$ follows from Eq. (12) upon substituting $\Psi_{\epsilon}(\mathbf{r}, t)$ [cf. Eq. (6)] in place of $\Psi(\mathbf{r}, t)$, i.e.,

$$
\left[\widetilde{\Phi}_{\epsilon}(\mathbf{r}, t)\right]^{*}=\left.\Phi_{\epsilon}(\mathbf{r},-t)\right|_{m \rightarrow-m, \mathbf{e} \rightarrow \mathbf{e}^{*}} .
$$

We note also a definition for $\widetilde{\Phi}_{\epsilon}(\mathbf{r}, t)$ that is equivalent to Eq. (14) [39]: the function $\Phi_{\epsilon}(\mathbf{r}, t)$ with its quasienergy complexconjugated (i.e., $\epsilon \rightarrow \epsilon^{*}$ ) and satisfying the ingoing-wave asymptotic boundary condition in the open (ionization) channels.

The normalization procedure for QQES wave functions, given by Eqs. (13) and (14), was suggested in Ref. [40] (see also Refs. [30,41] for additional discussions). As shown above, it is supported by the PT analysis. In addition, it represents a straightforward extension of the well-known "Zel'dovich normalization" for quasistationary (or resonance) states in time-independent problems (see Refs. $[42,43])$ to the case of time-dependent, QQES problems. Reference [42] emphasized the necessity of using dual functions in calculations of matrix elements within a basis of quasistationary states and indicated how to regularize the singular radial integrals that appear in these matrix elements and in the normalization integral (13), i.e., by introducing the regularization factor, $\exp \left(-\beta r^{2}\right)(\beta \rightarrow+0)$. [Recently, this
"Zel'dovich regularization" was employed in Refs. [44-46] for a number of time-independent problems involving singular integrals, including that of QQESs in a circularly polarized light field. (For this case the problem reduces to a stationary one in the reference frame rotating with frequency $\omega$ around the direction of the laser beam.)]

According to the normalization (13), the nonlinear susceptibilities (i.e., the response to a strong monochromatic perturbation) of an unstable system must be calculated by substituting $\left\langle\widetilde{\Psi}_{\epsilon}(t)\right|$ instead of $\langle\Psi(t)|$ in the definition (3) for the field-induced dipole moment, giving thus the definition for the "dual" dipole moment, $\widetilde{\mathbf{d}}(t)$,

$$
\begin{aligned}
\tilde{\mathbf{d}}(t) & =\left\langle\widetilde{\Psi}_{\epsilon}(t)|\hat{\mathbf{d}}| \Psi_{\epsilon}(t)\right\rangle=\left\langle\widetilde{\Phi}_{\epsilon}(t)|\hat{\mathbf{d}}| \Phi_{\epsilon}(t)\right\rangle \\
& =\frac{1}{2} \sum_{n=0}^{\infty}\left(\widetilde{\mathbf{d}}_{n \omega} e^{-i n \omega t}+\widetilde{\mathbf{d}}_{-n \omega} e^{i n \omega t}\right),
\end{aligned}
$$

where

$$
\tilde{\mathbf{d}}_{ \pm N \omega}=\frac{2}{T} \int_{0}^{T} d t e^{ \pm i N \omega t}\left\langle\widetilde{\Phi}_{\epsilon}(t)|\mathbf{d}| \Phi_{\epsilon}(t)\right\rangle .
$$

As defined in Eq. (15), $\widetilde{\mathbf{d}}(t)$ coincides with the standard definition (3) for a system without a continuous spectrum (i.e., neglecting ionization effects) and is consistent with the prescription (12) for taking account of ionization effects on the level of high-order PT corrections to the lowest-order PT result for nonlinear susceptibilities. Note that the necessity for using the "dual" form (15) for the field-induced dipole moment of a decaying system may be argued also in terms of "non-Hermitian quantum mechanics," in which dual functions are introduced as the "left" eigenfunctions of the Hamiltonian $\mathcal{H}^{\dagger^{*}}$, i.e., the transpose of $\mathcal{H}$ in the QQES equation (7) [47]. (This formulation was used in numerical HHG calculations for a one-dimensional inverted Gaussian potential [48] and for He [49,50].)

The nonperturbative HHG amplitude $\mathcal{A}_{N}\left(\mathbf{e}^{\prime}\right)$ in terms of $\tilde{\mathbf{d}}(t)$ is given by

$$
\mathcal{A}_{N}\left(\mathbf{e}^{\prime}\right)=\mathbf{e}^{\prime *} \cdot \tilde{\mathbf{d}}_{N \omega} .
$$

Complete information concerning the intensity and polarization properties of the $N$ th harmonic may be extracted from the amplitude (17), which describes dipole emission of radiation with frequency $N \omega$ and measured polarization $\mathbf{e}^{\prime}$. (While we do not discuss the general properties of the harmonics in the present paper, we note that appropriate QQES results for a ZRP model, including those for the elliptic dichroism effect, can be found in Refs. [51,52]. Also, for a review of polarization effects in HHG, see Ref. [13].) The total intensity, summed over the polarizations of the emitted photons, is proportional to $\left|\widetilde{\mathbf{d}}_{N \omega}\right|^{2}$ and is commonly described (see, e.g., Refs. $[20,22,40]$ ) by the harmonic rates $\mathcal{R}_{N}$, i.e., by the rates for dipole emission into the direction $\hat{\mathbf{k}}$ of the fundamental laser beam,

$$
\frac{d W_{N \omega, \hat{\mathbf{k}}}}{d \Omega_{\hat{\mathbf{k}}}} \equiv \mathcal{R}_{N}=\frac{(N \omega)^{3}}{8 \pi \hbar c^{3}}\left|\widetilde{\mathbf{d}}_{N \omega}\right|^{2} .
$$


As argued above, the fully quantum definition of the single-atom HHG amplitude, including proper account of laser field-induced Stark shifts and level widths, ultimately requires the use of a modified (dual) definition (15) of the dipole moment for a decaying system that differs from the commonly used definition (3) involving the expectation value of $\mathbf{d}$. Note that since $\operatorname{Im} \epsilon$ is small for not too strong fields, the matrix elements involved in $\mathbf{d}(t)$ and $\tilde{\mathbf{d}}(t)$ "feel" the divergence in the radial wave functions only at very large distances $r$ (beginning approximately from $r \sim r_{d}$ ). Thus numerical calculations of the HHG amplitude may give stable (convergent) results within a sphere $0 \leqslant r \leqslant R$, with $R<r_{d}$, using either the dual or the common definitions for the dipole moment. (For example, in the nonperturbative multiphoton regime, the difference between these two sets of results for the ground-state hydrogen atom is about $0.1 \%$ [39]. This difference increases substantially, however, with increasing $F$.) Nevertheless, a key difference between $\mathbf{d}(t)$ and $\tilde{\mathbf{d}}(t)$ is that the former is real-valued [as follows from Eq. (3), $\mathbf{d}_{-N \omega}=\mathbf{d}_{N \omega}^{*}$, while the dual dipole moment $\tilde{\mathbf{d}}(t)$ is complex, as follows from the relation

$$
\widetilde{\mathbf{d}}_{-N \omega}=\left.\widetilde{\mathbf{d}}_{N \omega}\right|_{m \rightarrow-m, \mathbf{e} \rightarrow \mathbf{e}^{*},}
$$

which may be verified by substituting $t \rightarrow-t$ in the integral over $t$ in Eq. (16) and employing the definition(14) for dual functions. In particular, $\tilde{\mathbf{d}}_{-N \omega}=\widetilde{\mathbf{d}}_{N \omega}$ when $\mathbf{F}(t)$ is linearly polarized, in which case $\mathbf{e}=\mathbf{e}^{*} \equiv \mathbf{e}_{z}$ and the results depend only on the modulus, $|m|$, of the azimuthal angular momentum quantum numbers with respect to the direction $\mathbf{e}_{z}$ of laser polarization.

\section{HHG AMPLITUDE IN TERMS OF THE COMPLEX QUASIENERGY}

Both accurate and approximate calculations of matrix elements for $\tilde{\mathbf{d}}_{N \omega}$ in Eq. (17) require knowledge of QQES wave functions over the entire coordinate space in order to evaluate the matrix element of $\widetilde{\mathbf{d}}(t)$ in Eq. (15). However, a number of approximate methods allow one to obtain these wave functions with reasonable accuracy only over a limited interval of $r$, mostly outside the atomic core. For these cases, it is therefore not possible to obtain accurate values either for $\widetilde{\mathbf{d}}_{N \omega}$ or for the proper normalization of $\widetilde{\Phi}_{\epsilon}(\mathbf{r}, t)$ according to Eq. (13). For this reason, we present below an alternative expression for the HHG amplitude (17), in terms of the complex quasienergy, that avoids the explicit involvement of QQES wave functions. The derivation of the appropriate expression provides also additional justification for the correctness of the definition of $\mathcal{A}_{N}\left(\mathbf{e}^{\prime}\right)$ in Eq. (17).

Our derivation is based on the Hellmann-Feynman theorem for quasistationary states [30]. We note first that for systems without a continuous spectrum (i.e., for quasienergy states having a real-valued, discrete quasienergy spectrum $\left.\epsilon_{n}\right)$, this theorem was introduced in Ref. [37] and has been employed in various applications (see, e.g., Ref. [53]). It has a form similar to that for time-independent Hamiltonians,

$$
\frac{\partial \epsilon_{n}(\lambda)}{\partial \lambda}=\frac{1}{T} \int_{0}^{T} d t\left\langle\Phi_{\epsilon_{n}}(\mathbf{r}, t)\left|\frac{\partial \hat{\mathcal{H}}(\mathbf{r}, t ; \lambda)}{\partial \lambda}\right| \Phi_{\epsilon_{n}}(\mathbf{r}, t)\right\rangle,
$$

where the "QES Hamiltonian" $\hat{\mathcal{H}}(\mathbf{r}, t ; \lambda)$ is defined by Eq. (7) and depends parametrically on some parameter $\lambda$. As argued in Ref. [30], the relation (20) holds also for QQESs, replacing $\left\langle\Phi_{\epsilon_{n}}(\mathbf{r}, t)\right|$ by the dual bra-vector $\left\langle\widetilde{\Phi}_{\epsilon}(\mathbf{r}, t)\right|$. In particular, choosing the parameter $\lambda$ in $\hat{\mathcal{H}}(\mathbf{r}, t ; \lambda)$ to be $\lambda=F \mathbf{e}^{*}$ $\equiv \mathbf{F}^{*}$, i.e., $\partial \hat{\mathcal{H}}(\mathbf{r}, t ; \lambda) / \partial \lambda=\partial V(\mathbf{r}, t) / \partial \mathbf{F}^{*}=-(1 / 2) \mathbf{d} \exp (i \omega t)$, one obtains [cf. Eqs. (1), (7), and (16):

$$
\frac{\partial \boldsymbol{\epsilon}}{\partial \mathbf{F}^{*}}=\frac{1}{T} \int_{0}^{T} d t\left\langle\tilde{\Phi}_{\epsilon}(\mathbf{r}, t)\left|\frac{\partial V(\mathbf{r}, t)}{\partial \mathbf{F}^{*}}\right| \Phi_{\epsilon}(\mathbf{r}, t)\right\rangle=-\frac{1}{4} \tilde{\mathbf{d}}_{\omega} .
$$

This equation is valid for an arbitrary $F$ and connects the Fourier-component of $\tilde{\mathbf{d}}(t)$ for the fundamental frequency $\omega$ with the complex quasienergy.

To obtain a relation similar to Eq. (21) for the dipole moment components corresponding to the harmonic frequency $\Omega, \widetilde{\mathbf{d}}_{N \omega}$, we add to the QQES Hamiltonian $\hat{\mathcal{H}}(\mathbf{r}, t)$ in Eq. (7) an interaction with a probe ("harmonic") field $\mathbf{F}_{h}(t)$ of amplitude $F_{h}, \quad V_{h}(\mathbf{r}, t)=-\mathbf{d} \cdot \mathbf{F}_{h}(t), \quad$ where $\quad \mathbf{F}_{h}(t)$ $=F_{h} \operatorname{Re}\left[\mathbf{e}^{\prime} \exp (-i \Omega t)\right]\left(\mathbf{e}^{\prime} \cdot \mathbf{e}^{\prime *}=1\right)$,

$$
\hat{\mathcal{H}}(\mathbf{r}, t) \rightarrow \hat{\mathcal{H}}(\mathbf{r}, t)=\hat{\mathcal{H}}(\mathbf{r}, t)+V_{h}(\mathbf{r}, t) .
$$

We assume that both fields propagate in the same direction, $\hat{\mathbf{k}}$, so that the vectors $\mathbf{F}(t)$ and $\mathbf{F}_{h}(t)$ lie in the plane perpendicular to $\hat{\mathbf{k}}$. Since the fields $\mathbf{F}(t)$ and $\mathbf{F}_{h}(t)$ have commensurable frequencies, $\Omega=N \omega$, the ordinary (single-frequency) QQES formalism continues to apply to this bichromatic field case. We denote by $\epsilon^{\prime}$ the quasienergy corresponding to the initial state $\psi_{0}(\mathbf{r})$ in the two-color field $\mathbf{F}(t)+\mathbf{F}_{h}(t)$. The Hellmann-Feynman theorem for the QQES $\Phi_{\epsilon^{\prime}}(\mathbf{r}, t)$ with $\lambda$ $=F_{h} \mathbf{e}^{\prime *} \equiv \mathbf{F}_{h}^{*}$ gives,

$$
\frac{\partial \boldsymbol{\epsilon}^{\prime}}{\partial \mathbf{F}_{h}^{*}}=\frac{1}{T} \int_{0}^{T} d t\left\langle\tilde{\Phi}_{\epsilon^{\prime}}(\mathbf{r}, t)\left|\frac{\partial V_{h}(\mathbf{r}, t)}{\partial \mathbf{F}_{h}^{*}}\right| \Phi_{\epsilon^{\prime}}(\mathbf{r}, t)\right\rangle=-\frac{1}{4} \widetilde{\mathbf{d}}_{\Omega}^{\prime},
$$

where

$$
\tilde{\mathbf{d}}_{\Omega}^{\prime}=\frac{2}{T} \int_{0}^{T} d t e^{i \Omega t}\left\langle\widetilde{\Phi}_{\epsilon^{\prime}}(\mathbf{r}, t)|\mathbf{d}| \Phi_{\epsilon^{\prime}}(\mathbf{r}, t)\right\rangle .
$$

Equations (23) and (24) are exact and valid even when both fields $\mathbf{F}(t)$ and $\mathbf{F}_{h}(t)$ are strong, so that in general $\tilde{\mathbf{d}}_{\Omega}^{\prime}$ depends nonlinearly on both $F$ and $F_{h}$. However, in the case of a weak (infinitesimal) harmonic field, $F_{h} \rightarrow 0, \tilde{\mathbf{d}}_{\Omega}^{\prime}$ in Eq. (23) reduces to the dual dipole moment component $\tilde{\mathbf{d}}_{\Omega}$ for $\mathbf{F}_{h}(t)=0$, which enters the HHG rate (18). Indeed, for this case we have $\epsilon^{\prime}=\epsilon+\Delta \epsilon$, where $\Delta \epsilon$ is linear in $F_{h}$ and, according to PT for QQESs, is given by the first-order (in $F_{h}$ ) PT correction to $\epsilon$, 


$$
\begin{aligned}
\Delta \epsilon & =\frac{1}{T} \int_{0}^{T} d t\left\langle\widetilde{\Phi}_{\epsilon}(\mathbf{r}, t)\left|V_{h}(\mathbf{r}, t)\right| \Phi_{\epsilon}(\mathbf{r}, t)\right\rangle \\
& =-\frac{F_{h}}{2 T} \int_{0}^{T} d t\left\langle\widetilde{\Phi}_{\epsilon}(\mathbf{r}, t)\left|e^{i \Omega t} \mathbf{e}^{\prime *} \cdot \mathbf{d}+e^{-i \Omega t} \mathbf{e}^{\prime} \cdot \mathbf{d}\right| \Phi_{\epsilon}(\mathbf{r}, t)\right\rangle .
\end{aligned}
$$

Equation (23) now gives the desired result for $\tilde{\mathbf{d}}_{N \omega}$ in terms of $\Delta \epsilon$ [cf. (16)],

$$
\tilde{\mathbf{d}}_{N \omega}=-4 \frac{\partial \Delta \epsilon}{\partial \mathbf{F}_{h}^{*}}=\frac{2}{T} \int_{0}^{T} d t e^{i N \omega t}\left\langle\widetilde{\Phi}_{\epsilon}(\mathbf{r}, t)|\mathbf{d}| \Phi_{\epsilon}(\mathbf{r}, t)\right\rangle,
$$

which is independent of $\mathbf{F}_{h}(t)$ and reduces to Eq. (21) for $N=1(\Omega=\omega)$.

For a linearly polarized field $\mathbf{F}(t)\left(\mathbf{e}=\mathbf{e}^{*} \equiv \mathbf{e}_{z}\right)$, the vector $\tilde{\mathbf{d}}_{N \omega}$ is proportional to $\mathbf{e}_{z}$ (since there are no other vectors in the problem),

$$
\tilde{\mathbf{d}}_{N \omega}=\widetilde{\chi}_{N}(\omega, F) \mathbf{e}_{z},
$$

where $\tilde{\chi}_{N}(\omega, F)$ is the generalized nonlinear susceptibility, which reduces to the usual nonlinear susceptibility, $\chi_{N}(N \omega)$ (cf. Sec. II B), in the lowest order in $F$ : $\widetilde{\chi}_{N}(\omega, F \rightarrow 0)$ $=\chi_{N}(N \omega) F^{N}$. According to Eq. (17), the HHG amplitude is given by

$$
\mathcal{A}_{N}=\mathbf{e}^{\prime *} \cdot \widetilde{\mathbf{d}}_{N \omega}=\widetilde{\chi}_{N}(\omega, F)\left(\mathbf{e}^{\prime *} \cdot \mathbf{e}_{z}\right),
$$

while the harmonic rate (18) reduces to

$$
\mathcal{R}_{N}=\frac{(N \omega)^{3}}{8 \pi \hbar c^{3}}\left|\widetilde{\chi}_{N}(\omega, F)\right|^{2} .
$$

Since the final result for $\widetilde{\mathbf{d}}_{N \omega}$ in Eq. (27) is independent of $\mathbf{F}_{h}(t)$, in calculating $\Delta \epsilon$ in Eq. (25) for the case of a linearly polarized laser field, $\mathbf{F}(t)$, it is sufficient to calculate only the linear in $F_{h}$ correction $\Delta \epsilon$ to the quasienergy in the linearly polarized two-color field $\widetilde{\mathbf{F}}(t)=\widetilde{F}(t) \mathbf{e}_{z}$, where $\widetilde{F}(t)=F \cos \omega t$ $+F_{h} \cos \Omega t(\Omega=N \omega)$.

Finally, we note that the definition (15) does not presume a direct connection [as in Eqs. (21) and (23)] between the Fourier components of $\tilde{\mathbf{d}}(t)$ and the complex quasienergy. Rather, our goal in introducing $\widetilde{\mathbf{d}}(t)$ was only to avoid both the divergences that occur in the commonly used definition (3) for the dipole moment in the strong field regime as well as the nonregularizable singularities that occur in high-order PT corrections to the nonlinear susceptibilities in the PT regime. Equations (21) and (23) may also be considered to be definitions of the Fourier components of a field-induced dipole moment in terms of the complex quasienergy, which is the eigenvalue of the Schrödinger equation for the QQES and is thus insensitive to the normalization of the QQES wave function. The remarkable coincidence of $\tilde{\mathbf{d}}_{\omega}$ and $\widetilde{\mathbf{d}}_{\Omega}^{\prime}$ in Eqs. (21) and (23) with those defined in terms of the dual functions in Eqs. (16) and (24) gives, apparently, a convincing justification for the consistency of redefining the dipole moment in Eq. (3) for a decaying system as in Eq. (15), i.e., using the dual functions (14). In addition, upon substituting the formal PT expansion (11) for $\epsilon$ into Eq. (21) and obtaining the expressions for $\beta_{2 n}(\omega)$ in terms of high-order PT matrix elements using Eq. (10), Eq. (21) gives the PT expansion for $\widetilde{\mathbf{d}}_{\omega}$ involving unambiguous, convergent expressions for the high-order PT corrections [i.e., high-order hyperpolarizabilities, $\left.\chi_{2 n-1}(\omega)\right]$ to the dynamic polarizability $\alpha(\omega)$ of the state $\psi_{0}(\mathbf{r})$. [Note that Ref. [29] used Eq. (21) to properly define the hyperpolarizability $\chi_{3}(\omega)$ at above-threshold frequencies, a case not requiring the dual function formalism.]

\section{CONNECTION WITH COMMON SEMIANALYTICAL METHODS FOR HHG CALCULATIONS}

In this section we connect the formulation in terms of the dual dipole moment to commonly used alternative approaches for HHG rates. The above considerations demonstrate that the term "dipole moment" for an atomic system interacting with an intense laser field (in which case ionization effects are important) has a conditional meaning. Its clearest physical interpretation is that $\mathbf{e}^{\prime^{*}} \cdot \widetilde{\mathbf{d}}_{N \omega}$ is the amplitude for conversion of $N$ laser photons into a high-order harmonic photon having polarization vector $\mathbf{e}^{\prime}$ and with the atom remaining in the field-free bound state $\psi_{0}(\mathbf{r})[30,51]$. In contrast, the expectation value of $\mathbf{d}$ for a decaying system is formally divergent and thus does not correspond to a physical observable. These divergences imply therefore that the dipole moment expectation value (3) cannot be used for $a b$ initio calculations of the atomic response to a strong monochromatic perturbation. Although the discussed divergences may be (erroneously) avoided in direct numerical calculations (e.g., by restricting the interval of integration over $r$ ) or may vanish within some approximations [such as the Keldysh approximation (KA), as discussed below], they must appear as nonregularizable singularities in any accurate analytical calculation of $\mathbf{d}(t)$ involving integrations of wave functions over the entire coordinate space. For example, the expectation value of $\mathbf{d}$ in Eq. (3) was used in detailed HHG calculations [20] starting from the exact QQES equations for a ZRP model [33]. The divergence of $\mathbf{d}_{N \omega}$ for $N=1$ is explicitly shown in Ref. [20] and, for higher $N$, the divergences were avoided in Ref. [20] by neglecting all but one of the Fourier-coefficients of the QQES wave function near the origin. This latter approximation is similar to the KA in HHG theory [27]. We note that the numerical results in Ref. [20], for the laser parameters that are considered there, are in good agreement with exact "dual" ZRP results [54] except for small values of $N$.

We emphasize that the dual function $\widetilde{\Phi}_{\epsilon}(\mathbf{r}, t)$ remains different from $\Phi_{\epsilon}(\mathbf{r}, t)$ even when the unperturbed bound state energy $E_{0}$ is employed in place of the complex quasienergy $\epsilon$ owing to the different (ingoing and outgoing spherical wave) boundary conditions for $\widetilde{\Phi}_{\epsilon=E_{0}}(\mathbf{r}, t)$ and $\Phi_{\epsilon=E_{0}}(\mathbf{r}, t)$ at large distances. In the approximation that $\epsilon=E_{0}$, the exact dual dipole moment (15) reduces to that in the " $S$-matrix approach" [see Eqs. (2.27)-(2.29) in Ref. [21]] and to that in Eq. (2.8) of Ref. [22]. Although the approaches used in Refs. $[21,22]$ (and also in the $S$-matrix formulation for the HHG process in Ref. [14]) fail to account for the laser field- 
induced level shift and width effects, in calculations of the matrix element of $\mathbf{d}$ in these references use is made of initial and final state wave functions that differ in the range of the temporal integrations and hence result nevertheless in a complex value of the dipole moment. Note that in approximating $\epsilon$ by $E_{0}$, the exact dual dipole moment results for the HHG amplitude reduce to the initial ("exact") results for the HHG amplitude in Refs. [14,21,22] (which contain exact Green functions of the atomic electron in a laser field). Note, however, that neither the $S$-matrix method nor the equivalent semianalytical method [22] has ever been used for practical HHG calculations beyond the simplest "strong field" approximation, in which the binding potential $U(\mathbf{r})$ is completely neglected in intermediate states of the active electron. This approximation is similar to the KA $[55,56]$ for the ionization amplitude. In particular, the approximate initial and final state wave functions used in actual calculations in Ref. [22] coincide exactly with the wave functions $\Phi_{\mathrm{KA}}(\mathbf{r}, t)$ and $\widetilde{\Phi}_{\mathrm{KA}}(\mathbf{r}, t)$ in the KA to the QQES [27]. (For the ZRP model, comparisons of exact QQES results with those in Refs. [21,22] can be found in Ref. [54]; see also Refs. [51,52] in which the first accurate calculation of HHG rates for a ZRP model were performed using the dual dipole moment within the QQES approach.)

Concerning the dipole moment expectation value $\mathbf{d}(t)$ in Eq. (3) in the $\left(\epsilon=E_{0}\right)$ wave function approximation, we note that it remains generally divergent, as in the case that the exact $\epsilon$ is used in Eq. (3). In particular, in Eq. (2.15) for $\mathbf{d}(t)$ in Ref. [21] the formal divergences exist in those terms corresponding to "continuum-continuum transitions" [i.e., the fourth term in Eq. (2.15)], within which nonregularizable singularities appear in the double temporal integral in the matrix element involving two exact time-dependent Green functions of the atomic electron in a laser field. Note that in the KA [or strong field approximation, i.e., replacing these exact Green functions by free-electron Green functions $G^{(V)}\left(\mathbf{r}, t ; \mathbf{r}^{\prime}, t^{\prime}\right)$ (cf. the Appendix)], the aforementioned singularities disappear and the KA result for the dipole moment expectation value, $\mathbf{d}^{\mathrm{KA}}(t)$ [cf. Eq. (2.16) in Ref. [21]], becomes finite in this approximation even with the inclusion of continuum-continuum terms. Moreover, by neglecting the continuum-continuum transitions this result reduces to that in the effective-dipole (or Lewenstein) model [19]. A comparison of results of this latter model with those in the strong field approximation version of the $S$-matrix approach is given in Ref. [21] for the polarization properties of harmonics produced by an elliptically polarized field within the ZRP model. In spite of the fact that both models agree quite well in many respects (for the laser parameters considered in Ref. [21]), there are instances in which the results are different, such as, e.g., for the rotation angle of the polarization ellipse of high harmonics with energies around the end of the plateau. A similar comparison of the magnitudes of HHG rates for the He atom is discussed in Ref. [14] (cf. Sec. II F 3): while for $N \gg 1$ the $S$ matrix and effective-dipole results agree very well (to within 1\%), for small $N$ the differences between the results increase to as much as $17 \%$ (for $N=5$ ).

In spite of the fact that in the Keldysh (or strong field) approximation the numerical differences between $S$-matrix

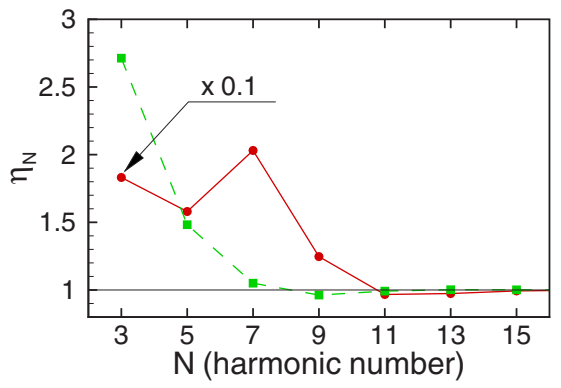

FIG. 1. (Color online) The ratio, $\eta_{N}=\left|\mathbf{d}_{N \omega}^{\mathrm{KA}} / \widetilde{\mathbf{d}}_{N \omega}^{\mathrm{KA}}\right|^{2}$, for a ZRP model $s$ state of energy $E_{0}$ for $F=0.2 F_{0}$ and $\omega=0.045 \omega_{0}$ (circles; solid line) and $0.098 \omega_{0}$ (squares; dashed line). $\omega_{0}=\left(\left|E_{0}\right| / \hbar\right)$ and $F_{0}=\sqrt{2 m\left|E_{0}\right|^{3}} /(|e| \hbar)$. Note that $\mathbf{d}_{N \omega}^{\mathrm{KA}}$ diverges for $N=1$ and for $\omega$ $=0.045 \omega_{0}$, the ratio for $N=3$ is divided by 10 .

and dipole moment expectation value results for HHG rates are small, the differences are nevertheless significant as they raise the question of how to interpret two different numerical values for the microscopic atomic parameter describing the emission of harmonic photons, both of which take into account the effects of the binding potential on the same level of accuracy, i.e., only in the bound state wave function $\psi_{0}(\mathbf{r})$. This problem has been known for about 10 years [21]. Since the question of the divergence of the apparently exact expression (3) for the dipole moment expectation value in the strong field regime did not attract much attention at the time, apparently in order to resolve this dilemma, the authors of Ref. [21] suggested that one consider the dipole moment expectation value as the atomic parameter relevant to the coherent emission of harmonics in the propagation of a laser pulse through an atomic medium, while one should consider the $S$-matrix result as describing the emission of harmonic photons by a single atom. Although this statement was criticized in Ref. [22], in which it was argued that "the theory of collective emission should be ultimately based on the proper description of a single atom process," some authors still apparently accept the rather surprising conception of a "duality" of microscopic quantum parameters relevant to the harmonic photon emission by a single atom on the one hand and by an ensemble of dynamically noninteracting atoms on the other (cf. Ref. [14]). Our considerations above give the underlying physical explanation of this surprising duality. Namely, the dipole moment expectation value in the KA, $\mathbf{d}^{\mathrm{KA}}(t)$, becomes finite only as a consequence of its neglect of binding potential effects in the exact result (3) for $\mathbf{d}(t)$, which otherwise becomes divergent (and thus physically meaningless) in the strong field regime, even in the $\left(\epsilon=E_{0}\right)$ approximation.

In order to illustrate explicitly the divergences in dipole moment expectation value results, in the Appendix we present these results in an analytic form for the exactly solvable ZRP model and compare them with the corresponding dual dipole moment results. For numerical comparisons, in Fig. 1 we compare KA dipole moment expectation value results with dual dipole moment results in the KA (which are equivalent to $S$-matrix results in the strong field approximation) for a ZRP model. Specifically, we present the ratio of $\left|\mathbf{d}_{N \omega}^{\mathrm{KA}}\right|^{2}$ to $\left|\widetilde{\mathbf{d}}_{N \omega}^{\mathrm{KA}}\right|^{2}$ (which equals the ratio of HHG rates) calcu- 
lated according to Eqs. (A12), (A14), (A13), and (A15) (at $k=k^{\prime}=0, f_{0}=\sqrt{2}$ and $\left.\epsilon=E_{0}=-1\right)$ at two different frequencies for the case of the bound ZRP model $s$ state. The ratio differs significantly from unity only in the region of low-order harmonics, up to the plateau onset (i.e., the extent of this region increases with decreasing $\omega$ ). The qualitative explanation of this result follows from our quantum interpretation [27] of plateau structures in HHG in terms of the Fourier components, $\Phi_{s}^{\mathrm{KA}}(\mathbf{r})$ [cf. Eq. (6)], of the KA wave function $\Phi_{\mathrm{KA}}(\mathbf{r}, t)$. In the strong-field regime, the Fourier component of the KA dual dipole moment,

$$
\tilde{\mathbf{d}}_{N \omega}^{\mathrm{KA}}=\sum_{s=-\infty}^{\infty}\left\langle\tilde{\Phi}_{s-N}^{\mathrm{KA}}|\mathbf{d}| \Phi_{s}^{\mathrm{KA}}\right\rangle,
$$

is well approximated by the two terms involving $\Phi_{0}^{\mathrm{KA}}(\mathbf{r})$ and $\widetilde{\Phi}_{0}^{\mathrm{KA}}(\mathbf{r})$ [which is equivalent to neglecting continuumcontinuum transitions [21] in the exact KA result (30)],

$$
\widetilde{\mathbf{d}}_{N \omega}^{\mathrm{KA}} \approx\left\langle\psi_{0}|\mathbf{d}| \Phi_{N}^{\mathrm{KA}}\right\rangle+\left\langle\widetilde{\Phi}_{-N}^{\mathrm{KA}}|\mathbf{d}| \psi_{0}\right\rangle
$$

where we have in addition approximated $\Phi_{0}^{\mathrm{KA}}(\mathbf{r})$ and $\widetilde{\Phi}_{0}^{\mathrm{KA}}(\mathbf{r})$ by $\psi_{0}(\mathbf{r})$. [Note that according to the definition (14) of the dual function, $\widetilde{\Phi}_{s}(\mathbf{r})=\Phi_{s}^{*}(\mathbf{r})$ for $s$ states.] As shown in Ref. [27], plateaus in HHG spectra originate from similar plateau structures in the spectrum of the harmonics $\Phi_{s}^{\mathrm{KA}}(\mathbf{r})$ with positive numbers $s$, beginning from $s \approx\left|E_{0}\right| /(\hbar \omega)$ up to $s$ $\approx\left(\left|E_{0}\right|+3.17 u_{p}\right) /(\hbar \omega)$. In this region the contribution of the last term in Eq. (31) is negligible, while for $N<\left|E_{0}\right| /(\hbar \omega)$ its contribution increases rapidly with decreasing $N$. (Within the three-step model [22], the smallness of this "time-reversed" term is interpreted in terms of the rescattering scenario $[16,18,57]$.) The Fourier-component $\mathbf{d}_{N \omega}^{\mathrm{KA}}$ of the dipole moment expectation value $\mathbf{d}^{\mathrm{KA}}(t)$ also has the form (31) upon substituting $\widetilde{\Phi}_{-N}^{\mathrm{KA}} \rightarrow \Phi_{-N}^{\mathrm{KA}}$ in the "time-reversed" term (which is equivalent to its complex conjugation). As discussed above, this substitution has no consequence for the plateau part of the HHG spectrum, but leads to inaccuracies of $\left|\mathbf{d}_{N \omega}^{\mathrm{KA}}\right|^{2}$ for low-order harmonics (cf. Fig. 1).

Therefore, in contrast to the KA version of the $S$-matrix result [which follows from the $a b$ initio result (15) for the dual dipole moment by (i) approximating $\epsilon=E_{0}$ and (ii) neglecting binding potential effects in intermediate states of the active electron], $\mathbf{d}^{\mathrm{KA}}(t)$ cannot be obtained unambiguously as the approximate version of a well-behaved, proper $a b$ initio definition of the dipole moment expectation value. Nevertheless, $\mathbf{d}^{\mathrm{KA}}(t)$ (or its reduced, effective-dipole version) has been employed for HHG analyses on an equal footing with results of the $S$-matrix approach and, generally, both methods yield comparable numerical results. However, if the differences between the dipole moment expectation values and the $S$-matrix results become significant (as, e.g., for the case of small $N$ in Fig. 1), then the latter results must be taken to be the physically meaningful ones.

\section{HHG RATES FOR THE CASE OF AN INITIAL STATE WITH NONZERO ANGULAR MOMENTUM}

For simplicity, in our considerations so far it has been assumed that the initial bound state $\psi_{0}(\mathbf{r})$ and the corresponding QQES $\Phi_{\epsilon}(\mathbf{r}, t)$ are nondegenerate, e.g., that $\psi_{0}(\mathbf{r})$ describes an $s$ state. For an initial state with nonzero angular momentum $l, \psi_{0}(\mathbf{r})=\varphi_{\kappa l m}(r) Y_{l m}(\hat{\mathbf{r}})$, the QQES approach becomes more complicated for the case of an elliptically polarized laser field, which mixes initially degenerate sublevels with different angular momentum projections $m$, so that $\psi_{0}(\mathbf{r})$ evolves in a laser field to $(2 l+1)$ Stark-split QQES substates, $\Phi_{\epsilon_{i}}(\mathbf{r}, t)(i=1, \ldots, 2 l+1)$, with generally different complex quasienergies $\epsilon_{i}$ [31]. The situation simplifies for the case of a linearly polarized field $\mathbf{F}(t)$, which we assume below. In this case the projection $m$ of the angular momentum on the direction of laser polarization, $\mathbf{e} \equiv \mathbf{e}_{z}$, is a conserved quantum number (as follows from the axial symmetry of the problem) and $\psi_{0}(\mathbf{r})$ splits into $(l+1)$ substates $\Phi_{\epsilon_{|m|}, m}(\mathbf{r}, t)$, with each of them (except for $m=0$ ) being doubly degenerate (in the sign of $m$ ).

The definition of HHG rates $\mathcal{R}_{N}$ for a degenerate initial state $\psi_{0}(\mathbf{r})$ requires a detailed explanation as we have not found a proper definition in the literature. Although we consider $\mathcal{R}_{N}$ as a single-atom, microscopic parameter, we have in mind the generation of coherent radiation of frequency $\Omega=N \omega$ by an ensemble of uncorrelated atoms having randomly oriented angular momenta and subjected to a laser field $\mathbf{F}(t)$. The intensity of this radiation should thus be proportional to the square of the atomic density, $\mathcal{N}$ (as is wellestablished experimentally). On the quantum, single-atom level, this means that the proper quantum atomic parameter relevant to this problem is given by the tensor of coherent scattering of harmonic photons $(\Omega)$ by an atom $(A)$ in an $(N+1)$-photon scattering process involving the absorption of $N$ laser photons $(\omega)$,

$$
A+N \omega \rightarrow A+\Omega,
$$

or, equivalently, by the cross section for this coherent scattering process. The coherent scattering of radiation is possible only if initial and final states of the atom are identical, i.e., have the same quantum numbers [58] (see also the pedagogical example for harmonic generation in Ref. [59]). For a nondegenerate $s$ state, the process (32) is always coherent [58], so that the rate (18) is the relevant quantity. For $l>0$, however, the single-atom HHG rate must be calculated similarly to the cross section of coherent light scattering, i.e., by averaging the "diagonal" partial rates $\mathcal{R}_{N}^{(m)}$ over $m$,

$$
\mathcal{R}_{N} \equiv \frac{1}{2 l+1} \sum_{m} \mathcal{R}_{N}^{(m)}=\frac{(N \omega)^{3}}{8 \pi \hbar c^{3}} \frac{1}{2 l+1} \sum_{m}\left|\widetilde{\mathbf{d}}_{N \omega}^{(|m|)}\right|^{2},
$$

where $\tilde{\mathbf{d}}_{N \omega}^{(|m|)}$ is given by the matrix element of $\mathbf{d}$ in Eq. (16) involving wave functions $\Phi_{\epsilon_{|m|}, m}(\mathbf{r}, t)$ and $\widetilde{\Phi}_{\epsilon_{|m|}, m}(\mathbf{r}, t)$ with the same quantum number $m$, i.e.,

$$
\tilde{\mathbf{d}}_{N \omega}^{(|m|)}=\frac{2}{T} \int_{0}^{T} d t e^{i N \omega t}\left\langle\widetilde{\Phi}_{\epsilon_{|m|}, m}(\mathbf{r}, t)|\mathbf{d}| \Phi_{\epsilon_{|m|}, m}(\mathbf{r}, t)\right\rangle .
$$


In order to calculate $\tilde{\mathbf{d}}_{N \omega}^{(|m|)}$ in terms of the complex quasienergy $\boldsymbol{\epsilon}_{|m|}$, we can still use the Hellmann-Feynman theorem (23) considering split states $\Phi_{\epsilon_{|m|}^{\prime}, m}(\mathbf{r}, t)$ in a twocolor (strong laser and weak harmonic) field $\mathbf{F}(t)+\mathbf{F}_{h}(t)$ as single states. (The Hellmann-Feynman theorem for quasidegenerate or degenerate QQESs may be formulated similarly to that applicable to degenerate time-independent problems [60].) Following the transition from Eq. (23) to Eq. (26), one obtains

$$
\widetilde{\mathbf{d}}_{N \omega}^{(|m|)}=-4 \frac{\partial \Delta \boldsymbol{\epsilon}_{|m|}}{\partial \mathbf{F}_{h}^{*}},
$$

where $\Delta \epsilon_{|m|}\left(\Delta \epsilon_{|m|} \equiv \epsilon_{|m|}^{\prime}-\epsilon_{|m|}\right)$ is linear in $F_{h}$ and given by Eq. (25) in which $\widetilde{\Phi}_{\epsilon}(\mathbf{r}, t) \rightarrow \widetilde{\Phi}_{\epsilon_{|m|}, m}(\mathbf{r}, t)$. As a result, the Fourier-component $\tilde{\mathbf{d}}_{N \omega}^{(|m|)}$ calculated according to Eq. (35) is equivalent to that in Eq. (34). Since we consider a linearly polarized field $\mathbf{F}(t) \quad\left(\mathbf{e}=\mathbf{e}^{*} \equiv \mathbf{e}_{z}\right)$, the vector $\tilde{\mathbf{d}}_{N \omega}^{(|m|)}$ and the HHG amplitude $\mathcal{A}_{N}^{(|m|)}$ may be expressed in terms of the generalized nonlinear susceptibility $\tilde{\chi}_{N}^{(|m|)}(\omega, F)$ analogously to Eqs. (27) and (28),

$$
\begin{gathered}
\tilde{\mathbf{d}}_{N \omega}^{(|m|)}=\tilde{\chi}_{N}^{(|m|)}(\omega, F) \mathbf{e}_{z}, \\
\mathcal{A}_{N}^{(|m|)}=\tilde{\chi}_{N}^{(|m|)}(\omega, F)\left(\mathbf{e}^{\prime *} \cdot \mathbf{e}_{z}\right),
\end{gathered}
$$

so that the harmonic rate $(33)$ reduces to

$$
\mathcal{R}_{N}=\frac{(N \omega)^{3}}{8 \pi \hbar c^{3}} \frac{1}{2 l+1} \sum_{m}\left|\tilde{\chi}_{N}^{(|m|)}(\omega, F)\right|^{2} .
$$

As discussed below Eq. (29), in actual calculations of $\Delta \epsilon_{|m|}$ in Eq. (25) it is sufficient to consider only the linearly polarized two-color field $\widetilde{\mathbf{F}}(t)=\widetilde{F}(t) \mathbf{e}_{z}$, where $\widetilde{F}(t)=F \cos \omega t$ $+F_{h} \cos \Omega t$.

The definitions (33) and (38) differ from the definition of the cross section for spontaneous light scattering, or of the spontaneous dipole emission rate, in that the latter two involve averaging over the initial $(m)$ and summing over the final $\left(m^{\prime}\right)$ state magnetic quantum numbers, which in general differ, $m^{\prime}=m, m \pm 1$. Besides possible numerical differences between the HHG rates (33) and (38) and those defined for the case of a degenerate bound state $\psi_{0}(\mathbf{r})$ as the rates for spontaneous dipole emission, $\mathcal{R}_{N}^{s p}$, the most important difference is in the polarization properties of harmonic (as well as spontaneously emitted) photons. In particular, according to Eqs. (33) and (38), harmonics generated by a linearly polarized light field have the same (linear) polarization (as for the case of $s$ states), while the spontaneously emitted photons may be elliptically polarized (for transitions with $m^{\prime}=m \pm 1$ ). [The definition $\mathcal{R}_{N}^{s p}$ of the harmonic rates for a $p$ state $\psi_{0}(\mathbf{r})$ is assumed in Refs. $[23,61]$. Therefore, as discussed in these references, generation of elliptically polarized harmonics by atoms with nonzero angular momenta subjected to a linearly polarized pump field may be observed only as a weak (incoherent) spontaneous emission of photons of frequency $N \omega$, whose intensity is linear in $\mathcal{N}$.]

\section{CONCLUDING REMARKS}

The main result of this paper is the development of a wave-function-independent theoretical formulation of the HHG amplitude in terms of the complex quasienergy of a system. Based on the Hellmann-Feynman theorem for QQESs, in Sec. III we have formulated the HHG amplitude in terms of the complex quasienergy of a bound electron in a bichromatic (strong laser and weak harmonic) field. The amplitude for generation of the $N$ th harmonic can be defined in terms of the generalized nonlinear susceptibility $\widetilde{\chi}_{N}(\omega, F)$ of an atomic system subjected to both a strong field $\mathbf{F}(t)$ of frequency $\omega$ and a probe (harmonic) field of frequency $\Omega$ $=N \omega$. This susceptibility represents the generalization of the well-known intensity-independent (lowest-order PT) susceptibility $\chi_{N}(N \omega)$ to the case of a strong, non-perturbative laser field. Its expansion in $F$ inside the range of convergence of the PT series yields an infinite set of high-order nonlinear susceptibilities $\chi_{N+2 k}(N \omega)$, which are common in traditional ("perturbative") nonlinear optics (cf. Sec. II A). Our result for the HHG amplitude in terms of $\widetilde{\chi}_{N}(\omega, F)$ is equivalent to its definition in terms of the dual dipole moment, thereby giving additional justification for using dual QQES wave functions for calculating the quantum, single-atom response to a strong monochromatic perturbation that takes proper account of the accompanying ionization processes. As discussed in Sec. II, these results permit a clear and unambiguous way to avoid the nonregularizable singularities that appear in both weak-field (PT) and strong-field (nonperturbative) regimes of laser-atom interactions when using quantities that are only well-defined for a stable quantum system (such as the expectation value of the dipole operator) to describe the nonlinear phenomena that occur simultaneously with multiphoton above-threshold ionization. Also, these fully quantum, exact results, which are valid for a wide range of laser parameters $F$ and $\omega$, allow one to establish quantitatively the accuracy of common approximate (e.g., strong-field, low-frequency) results for the HHG amplitude.

In addition to the utility of our formulation for analyzing formal aspects of HHG theory, another advantage of formulating the HHG amplitude in terms of the complex quasienergy is that one avoids the necessity of knowing the explicit form of the QQES (as well as the initial bound state) wave functions in practical calculations of HHG rates. Indeed, in general, the complex quasienergy $\epsilon$ can be obtained as the eigenvalue of equations (7) or (8). Its determination does not require complete knowledge of the wave function $\Phi_{\epsilon}(\mathbf{r}, t)$ corresponding to this eigenvalue nor on the normalization of this function. This fact allows for practical calculations of HHG rates even for cases when the exact form of $\Phi_{\epsilon}(\mathbf{r}, t)$ over the entire space is unknown so that the straightforward calculation of the matrix element for $\tilde{\mathbf{d}}(t)$ in Eq. (15) is impossible. This very situation occurs in the time-dependent effective range (TDER) theory of strong laser processes [62], in which the QQES wave function $\Phi_{\epsilon}(\mathbf{r}, t)$ of a weakly bound electron is known only outside a short-range atomic core. The complex quasienergy, however, may be obtained independently of $\Phi_{\epsilon}(\mathbf{r}, t)$, as the eigenvalue of a onedimensional integro-differential equation for a periodic func- 
tion of time. The self-consistent, essentially analytical theory of HHG within the TDER theory is developed in the next paper [24] and is employed there to analyze quantitatively harmonic generation by negative ions.

\section{ACKNOWLEDGMENTS}

A number of valuable comments that have improved this paper were provided to the authors by our colleague, V. N. Ostrovsky, who passed away recently. This work was supported in part by RFBR Grant No. 07-02-00574 and by NSF Grant No. PHY-0601196. Two of the authors (M.V.F. and A.V.F.) acknowledge the support of the "Dynasty" Foundation, Grants of the President of the Russian Federation No. MK-1075.2005.2 and No. MK-8715.2006.2, and CRDF and BRHE grants for young scientists, Grants No. Y2-P-10-03 and No. Y2-P-10-06. One of the authors (A.F.S.) gratefully acknowledges partial support of the Alexander von Humboldt Stiftung and the Max-Planck-Institut für Quantenoptik (MPQ) as well as the hospitality of the MPQ, where part of this paper was prepared.

\section{APPENDIX: EXPLICIT FORM OF $\tilde{\mathrm{d}}(t)$ AND d $(t)$ FOR A ZRP MODEL}

We present here explicit expressions for the dipole moment expectation value (3) and dual dipole moment (15) for the case of a weakly bound electron in the $s$ state of energy $E_{0}$ of a ZRP. This model allows an essentially exact analytical treatment of the QQES problem [33]. In this appendix we use scaled units: energies are measured in units of $\left|E_{0}\right|$ and field amplitudes $F$ in units of $F_{0}=\sqrt{2 m\left|E_{0}\right|^{3}} /(|e| \hbar)$. The exact ZRP QQES wave function and its dual function [see Eqs. (8) and (14)] have the following form [34]:

$$
\begin{gathered}
\Phi_{\epsilon}(\mathbf{r}, t)=-\sqrt{4 \pi} \int_{0}^{\infty} d \tau e^{i \epsilon \tau} G^{(V)}(\mathbf{r}, t ; 0, t-\tau) f(t-\tau), \\
\widetilde{\Phi}_{\epsilon}^{*}(\mathbf{r}, t)=\sqrt{4 \pi} \int_{-\infty}^{0} d \tau^{\prime} e^{-i \epsilon \tau^{\prime}} G^{(V)}\left(0, t-\tau^{\prime} ; \mathbf{r}, t\right) f\left(\tau^{\prime}-t\right),
\end{gathered}
$$

where $\epsilon$ is the complex quasienergy; $f(t)=\Sigma_{k} f_{k} e^{-2 i k \omega t}$ is a periodic function that originates from the boundary condition for $\Phi_{\epsilon}(\mathbf{r}, t)$ at the origin [33]. The Fourier coefficients $f_{k}$ are normalized so that Eq. (13) is satisfied (for an explicit form of the normalization factor see, e.g., Appendix A in Ref. [34]); $G^{(V)}\left(\mathbf{r}, t, \mathbf{r}^{\prime}, t^{\prime}\right)$ is the (retarded) Green function for a free electron in a linearly polarized field $\mathbf{F}(t)=\mathbf{e}_{z} F \cos \omega t$, which we express in Feynman's form in terms of the classical action, $S\left(\mathbf{r}, t, \mathbf{r}^{\prime}, t^{\prime}\right)$,

$$
G^{(V)}\left(\mathbf{r}, t, \mathbf{r}^{\prime}, t^{\prime}\right)=-\frac{i \Theta\left(t-t^{\prime}\right)}{\left[4 \pi i\left(t-t^{\prime}\right)\right]^{3 / 2}} e^{i S_{c l}\left(\mathbf{r}, t, \mathbf{r}^{\prime}, t^{\prime}\right)},
$$

where $\Theta(x)$ is the Heaviside function,

$$
\begin{aligned}
S_{c l}\left(\mathbf{r}, t, \mathbf{r}^{\prime}, t^{\prime}\right)= & \frac{\left(\mathbf{r}-\mathbf{r}^{\prime}\right)^{2}}{4\left(t-t^{\prime}\right)}-\frac{\left(\mathbf{r}-\mathbf{r}^{\prime}\right)}{\omega^{2}\left(t-t^{\prime}\right)}\left[\mathbf{F}(t)-\mathbf{F}\left(t^{\prime}\right)\right] \\
& +\frac{\mathbf{r}}{\omega^{2}} \frac{\partial}{\partial t} \mathbf{F}(t)-\frac{\mathbf{r}^{\prime}}{\omega^{2}} \frac{\partial}{\partial t^{\prime}} \mathbf{F}\left(t^{\prime}\right)+S_{c l}\left(t, t^{\prime}\right),
\end{aligned}
$$

$$
\begin{aligned}
S_{c l}\left(t, t^{\prime}\right) \equiv & S_{c l}\left(\mathbf{r}=0, t, \mathbf{r}^{\prime}=0, t^{\prime}\right) \\
= & -\frac{u_{p}}{\omega}\left[\omega\left(t-t^{\prime}\right)\left(1-\frac{4 \sin ^{2}\left[\omega\left(t-t^{\prime}\right) / 2\right]}{\left[\omega\left(t-t^{\prime}\right)\right]^{2}}\right)\right. \\
& -\cos \omega\left(t+t^{\prime}\right)\left(\sin \omega\left(t-t^{\prime}\right)\right. \\
& \left.\left.-\frac{4 \sin ^{2}\left[\omega\left(t-t^{\prime}\right) / 2\right]}{\omega\left(t-t^{\prime}\right)}\right)\right] .
\end{aligned}
$$

It follows from Eqs. (A1) and (A2) and our use of the Feynman form for $G^{(V)}\left(\mathbf{r}, t ; \mathbf{r}^{\prime}, t^{\prime}\right)$, that the spatial integrals in the matrix elements (3) and (15) have Gaussian integrands and thus may be evaluated analytically. These spatial integrations are easily performed using the following equation for the case of $\tilde{\mathbf{d}}(t)$ :

$$
\begin{aligned}
\int d \mathbf{r} & G^{(V)}\left(0, t-\tau^{\prime} ; \mathbf{r}, t\right) \mathbf{r} G^{(V)}(\mathbf{r}, t ; 0, t-\tau) \\
= & \frac{2 i}{\omega^{2}\left(\tau^{\prime}-\tau\right)}\left\{\tau\left[\mathbf{F}\left(t-\tau^{\prime}\right)-\mathbf{F}(t)\right]-\tau^{\prime}[\mathbf{F}(t-\tau)-\mathbf{F}(t)]\right\} \\
& \times G^{(V)}\left(0, t-\tau^{\prime} ; 0, t-\tau\right)
\end{aligned}
$$

which is valid for $\left(t-\tau^{\prime}\right) \geqslant t \geqslant(t-\tau)$ [cf. Eqs. (A1) and (A2)]; and Eq. (B4) in Ref. [20] [involving the "homogeneous" Volkov propagators $\left.G^{(E)}\left(\mathbf{r} t, \mathbf{r}^{\prime} t^{\prime}\right)\right]$ for $\mathbf{d}(t)$. Thus, the problem reduces to the evaluation of double temporal integrals having similar integrands but different integration limits [for $\mathbf{d}(t)$, we use the " $\left(\epsilon=E_{0}\right)$ approximation," cf. Sec. IV],

$$
\begin{aligned}
\mathbf{d}(t)= & \frac{2 i \mathbf{e}_{z}}{\sqrt{\pi i}} \int_{0}^{\infty} d \tau \int_{0}^{\infty} d \tau^{\prime} \frac{e^{i S\left(0, t-\tau^{\prime} ; 0, t-\tau\right)}}{\left(\tau^{\prime}-\tau\right)^{5 / 2}} f(t-\tau) f^{*}\left(t-\tau^{\prime}\right) \\
& \times \mathcal{R}\left(t ; \tau, \tau^{\prime}\right) e^{i E_{0}\left(\tau-\tau^{\prime}\right)}
\end{aligned}
$$

$$
\begin{aligned}
\tilde{\mathbf{d}}(t)= & -\frac{2 i \mathbf{e}_{z}}{\sqrt{\pi i}} \int_{0}^{\infty} d \tau \int_{-\infty}^{0} d \tau^{\prime} \frac{e^{i S\left(0, t-\tau^{\prime} ; 0, t-\tau\right)}}{\left(\tau^{\prime}-\tau\right)^{5 / 2}} f(t-\tau) f\left(\tau^{\prime}-t\right) \\
& \times \mathcal{R}\left(t ; \tau, \tau^{\prime}\right) e^{i \epsilon\left(\tau-\tau^{\prime}\right)}
\end{aligned}
$$

where

$$
\begin{aligned}
\mathcal{R}\left(t ; \tau, \tau^{\prime}\right)= & \frac{F}{\omega^{2}}\left\{\tau^{\prime} \sin (\omega \tau / 2) \sin [\omega(t-\tau / 2)]\right. \\
& \left.-\tau \sin \left(\omega \tau^{\prime} / 2\right) \sin \left[\omega\left(t-\tau^{\prime} / 2\right)\right]\right\} .
\end{aligned}
$$

For further integrations, we introduce new variables in Eq. (A7) for $\mathbf{d}(t)$, 
$\zeta=\tau+\tau^{\prime}, \quad \xi=\tau^{\prime}-\tau, \quad$ where $-\infty<\xi<\infty, \quad|\xi| \leqslant \zeta<\infty$,

and in Eq. (A8) for $\tilde{\mathbf{d}}(t)$,

$\zeta=\tau+\tau^{\prime}, \quad \xi=\tau-\tau^{\prime}, \quad$ where $0 \leqslant \xi<\infty, \quad-\xi \leqslant \zeta \leqslant \xi$,

which correspond to the rotation of the $\left(\tau, \tau^{\prime}\right)$-plane by $45^{\circ}$ [clockwise for $\tilde{\mathbf{d}}(t)$ and counterclockwise for $\mathbf{d}(t)$ ]. In the new variables, the integration over $\zeta$ is carried out analytically, where for $\mathbf{d}(t)$, the standard substitution, $\alpha \rightarrow \alpha+i 0$, is used for regularization of singular integrals of the form $\int_{|\xi|}^{\infty} \zeta^{m} e^{i \alpha \zeta} d \zeta$. As a result, the Fourier components of $\mathbf{d}(t)$ and $\tilde{\mathbf{d}}(t)$ in Eqs. (3) and (15) may be expressed in terms of onedimensional integrals over $\phi \equiv \omega \xi / 2$ [cf. Eq. (3.14) for $\mathbf{d}_{n \omega}$ in Ref. [20]],

$$
\begin{aligned}
& \mathbf{d}_{n \omega}=\chi_{n}(\omega, F) \mathbf{e}_{z}, \quad \chi_{n}(\omega, F)=\sum_{k, k^{\prime}} f_{k}^{*} f_{k^{\prime}} \chi_{n ; k, k^{\prime}}, \\
& \tilde{\mathbf{d}}_{n \omega}=\tilde{\chi}_{n}(\omega, F) \mathbf{e}_{z}, \quad \tilde{\chi}_{n}(\omega, F)=\sum_{k, k^{\prime}} f_{k} f_{k^{\prime}} \tilde{\chi}_{n ; k, k^{\prime}},
\end{aligned}
$$

where $n$ (the harmonic number) is a positive odd integer, $n=1,3, \ldots$,

$$
\begin{aligned}
\chi_{n ; k, k^{\prime}}= & \mathcal{C}_{0} \int_{-\infty}^{\infty} \frac{d \phi}{\phi^{3 / 2}} e^{-2 i\left(E_{0} / \omega+k+k^{\prime}\right) \phi+i \lambda(\phi)} \\
& \times\left[r_{-}(\phi) \mathcal{J}_{-}(\phi)+i r_{+}(\phi) \mathcal{J}_{+}(\phi)\right], \\
\tilde{\chi}_{n ; k, k^{\prime}}= & \mathcal{C}_{0} \int_{0}^{\infty} \frac{d \phi}{\phi^{3 / 2}} e^{2 i\left(\epsilon / \omega+k+k^{\prime}\right) \phi-i \lambda(\phi)} \\
& \times\left[j_{-}(\phi) \mathcal{J}_{-}(\phi)-i j_{+}(\phi) \mathcal{J}_{+}(\phi)\right],
\end{aligned}
$$

where $\mathcal{J}_{ \pm}(\phi)$ is shorthand for the Bessel function, $\mathcal{J}_{ \pm}(\phi)$ $=J_{k-k^{\prime}+(n \pm 1) / 2}[z(\phi)]$,

$$
\begin{gathered}
\lambda(\phi)=\frac{2 u_{p}}{\omega}\left(\phi-\frac{\sin ^{2} \phi}{\phi}\right), \\
z(\phi)=\frac{2 u_{p}}{\omega} \sin \phi\left(\cos \phi-\frac{\sin \phi}{\phi}\right), \\
r_{ \pm}(\phi)=\frac{\sin \phi}{\phi} e^{i n|\phi|}-\frac{n}{n \pm 1} e^{i(n \pm 1)|\phi|} \\
j_{ \pm}(\phi)=\operatorname{Im}\left\{r_{ \pm}(\phi)\right\}=\frac{\sin \phi \sin (n \phi)}{\phi}-\frac{n \sin [(n \pm 1) \phi]}{n \pm 1},
\end{gathered}
$$

$$
\mathcal{C}_{0}=\frac{2 i^{k-k^{\prime}+n / 2}}{n^{2}} \sqrt{\frac{u_{p}}{\pi \omega^{3}}}, \quad u_{p}=\frac{F^{2}}{2 \omega^{2}} .
$$

Simple inspection of the integral in Eq. (A14) shows that the generalized nonlinear susceptibility $\chi_{n}(\omega, F)$ in Eq. (A12) for Fourier-component $\mathbf{d}_{n \omega}$ is generally divergent for any $n$. For $n=1$, it is obvious owing to the divergence of $r_{-}(\phi)$ in Eq. (A18), which appears as a result of the integration in $\mathbf{d}(t)$ over $\zeta$. For $n>1$, the nonintegrable singularity at small $\phi, \sim \phi^{-3 / 2}$, appears in the terms $\chi_{n ; k, k^{\prime}}$ with $k^{\prime}=k$ $+(n \pm 1) / 2$. Indeed, for small $\phi$, we have $r_{ \pm}(\phi) \approx[(2 \pm n) /(1$ $-n)], z(\phi) \sim \phi^{3}$, and $\mathcal{J}_{ \pm}(\phi) \approx 1$ for $k^{\prime}=k+(n \pm 1) / 2$ since $\left.J_{0}[z(\phi)] \approx 1\right\}$, so that the integrand in Eq. (A14) behaves as $\phi^{-3 / 2}$ at $|\phi| \rightarrow 0$. In contrast, for the dual dipole moment (A13), the integral in Eq. (A15) is finite for any $n, k$, and $k^{\prime}$, since $j_{ \pm}(\phi) \sim \phi^{3}$ and the integrand has a smooth behavior [e.g., $\sim \phi^{3 / 2}$ for $k^{\prime}=k+(n \pm 1) / 2$ ] at $\phi \approx 0$.

Mathematically, the divergence of $\mathbf{d}(t)$ stems from the difference between the integration limits for $\tau^{\prime}$ in Eqs. (A7) and (A8) and, as a consequence, between $\zeta$ and $\xi$ in Eqs. (A10) and (A11). Physically, this difference originates from the different asymptotic behaviors of $\Phi_{\epsilon}^{*}(\mathbf{r}, t)$ and $\widetilde{\Phi}_{\epsilon}^{*}(\mathbf{r}, t)$ at large distances: the latter behaves asymptotically as an outgoing wave, as does $\Phi_{\epsilon}(\mathbf{r}, t)$, so that the integrand in $\left\langle\widetilde{\Phi}_{\epsilon}(t)|\mathbf{d}| \Phi_{\epsilon}(t)\right\rangle$ contains oscillatory functions at $|\mathbf{r}| \rightarrow \infty$ and the spatial integral for $\tilde{\mathbf{d}}(t)$ is regularizable; $\Phi_{\epsilon}^{*}(\mathbf{r}, t)$ behaves asymptotically, in contrast to $\Phi_{\epsilon}(\mathbf{r}, t)$, as an ingoing wave, so that the oscillatory (at $|\mathbf{r}| \rightarrow \infty)$ terms cancel in the integrand of $\left\langle\Phi_{\epsilon}(t)|\mathbf{d}| \Phi_{\epsilon}(t)\right\rangle$ and the result for $\mathbf{d}(t)$ diverges. This result is general and does not depend upon the specific forms of $\Phi_{\epsilon}(\mathbf{r}, t)$ and $\widetilde{\Phi}_{\epsilon}^{*}(\mathbf{r}, t)$ in Eqs. (A1) and (A2) for the ZRP model.

For the ZRP model, the KA [in which, as discussed in Sec. IV, $\tilde{\mathbf{d}}(t)$ relates to the $S$-matrix result and $\mathbf{d}(t)$ to the dipole moment expectation value, $\left.\mathbf{d}^{\mathrm{KA}}(t)\right]$ corresponds to neglecting all coefficients $f_{k}$ except $f_{0}=\sqrt{2}$, i.e., approximating $f(t)=\sqrt{2}$ and $\epsilon=E_{0}$ [33]. In this approximation, $\tilde{\mathbf{d}}_{n \omega}$ in Eq. (A13) is determined by $\widetilde{\chi}_{n}^{\mathrm{KA}}(\omega, F)=\widetilde{\chi}_{n ; 0,0}$, substituting $\epsilon=E_{0}$ in the exponent in Eq. (A15), while $\mathbf{d}_{n \omega}^{\mathrm{KA}}$ also becomes finite (except for $n=1$ ) and the corresponding susceptibility, $\chi_{n}^{\mathrm{KA}}(\omega, F)$, is given by $\chi_{n ; 0,0}$ in Eq. (A14). This unexpected finiteness of $\mathbf{d}^{\mathrm{KA}}(t)$ is in some sense fortuitous and is a consequence of the unique relation (B4) in Ref. [20] [which is similar to Eq. (A6)], which occurs only for the free electron (Volkov) propagator $G^{(V)}\left(\mathbf{r}, t ; \mathbf{r}^{\prime}, t^{\prime}\right)$ and is not valid for the Green function of a bound (atomic) electron in a laser field. Finally, we note that our result for $\mathbf{d}_{n \omega}^{\mathrm{KA}}=\chi_{n}^{\mathrm{KA}}(\omega, F) \mathbf{e}_{z}$ is equivalent to that in Eq. (4.1) of Ref. [20] which was used there in actual numerical calculations of HHG rates for a ZRP model. 
[1] A. McPherson, G. Gibson, H. Jara, U. Johann, T. S. Luk, I. A. McIntyre, K. Boyer, and C. K. Rhodes, J. Opt. Soc. Am. B 4, 595 (1987).

[2] M. Ferray, A. L'Huillier, X. F. Li, L. A. Lompré, G. Mainfray, and C. Manus, J. Phys. B 21, L31 (1988).

[3] Z. Chang, A. Rundquist, H. Wang, M. M. Murnane, and H. C. Kapteyn, Phys. Rev. Lett. 79, 2967 (1997).

[4] C. Spielmann, N. H. Burnett, S. Sartania, R. Koppitsch, M. Schnürer, C. Kan, M. Lenzner, P. Wobrauschedk, and F. Krausz, Science 278, 661 (1997).

[5] Y. Nabekawa, H. Hasegawa, E. J. Takahashi, and K. Midorikawa, Phys. Rev. Lett. 94, 043001 (2005).

[6] P. Agostini and L. F. DiMauro, Rep. Prog. Phys. 67, 813 (2004).

[7] P. M. Paul, E. S. Toma, P. Breger, G. Mullot, F. Augé, P. Balcou, H. G. Muller, and P. Agostini, Science 292, 1689 (2001).

[8] M. Hentschel, R. Kienberger, C. Spielmann, G. A. Reider, N. Milosevic, T. Brabec, P. B. Corkum, U. Heinzmann, M. Drescher, and F. Krausz, Nature (London) 414, 509 (2001).

[9] R. Kienberger, E. Goulielmakis, M. Uiberacker, A. Baltuska, V. Yakovlev, F. Bammer, A. Scrinzi, T. Westerwalbesich, U. Kleineberg, U. Heinzmann, M. Drescher, and F. Krausz, Nature (London) 427, 817 (2004).

[10] M. Protopapas, C. H. Keitel, and P. L. Knight, Rep. Prog. Phys. 60, 389 (1997).

[11] P. Salières, A. L'Huillier, P. Antoine, and M. Lewenstein, Adv. At., Mol., Opt. Phys. 4, 83 (1999).

[12] T. Brabec and F. Krausz, Rev. Mod. Phys. 72, 545 (2000).

[13] W. Becker, F. Grabson, R. Kopold, D. B. Milošević, G. G. Paulus, and H. Walther, Adv. At., Mol., Opt. Phys. 48, 35 (2002).

[14] D. B. Milošević and F. Ehlotzky, Adv. At., Mol., Opt. Phys. 49, 373 (2003)

[15] J. L. Krause, K. J. Schafer, and K. C. Kulander, Phys. Rev. Lett. 68, 3535 (1992).

[16] K. C. Kulander, K. J. Schafer, and J. L. Krause, in SuperIntense Laser-Atom Physics, edited by B. Piraux, A. L'Huillier, and K. Rzążewski (Plenum, New York, 1993), pp. 95-110.

[17] K. J. Schafer, B. Yang, L. F. DiMauro, and K. C. Kulander, Phys. Rev. Lett. 70, 1599 (1993).

[18] P. B. Corkum, Phys. Rev. Lett. 71, 1994 (1993).

[19] M. Lewenstein, P. Balcou, M. Yu. Ivanov, A. L'Huillier, and P. B. Corkum, Phys. Rev. A 49, 2117 (1994).

[20] W. Becker, S. Long, and J. K. McIver, Phys. Rev. A 50, 1540 (1994).

[21] W. Becker, A. Lohr, M. Kleber, and M. Lewenstein, Phys. Rev. A 56, 645 (1997).

[22] M. Yu. Kuchiev and V. N. Ostrovsky, Phys. Rev. A 60, 3111 (1999).

[23] V. N. Ostrovsky, J. Phys. B 38, 4399 (2005).

[24] M. V. Frolov, A. V. Flegel, N. L. Manakov, and A. F. Starace, Phys. Rev. A 75, 063408 (2007), following paper.

[25] H. Hellmann, Einführung in die Quantumchemie (Deuticke, Leipzig, Vienna, 1937).

[26] R. P. Feynman, Phys. Rev. 56, 340 (1939).

[27] M. V. Frolov, A. A. Khuskivadze, N. L. Manakov, and A. F. Starace, J. Phys. B 39, S283 (2006).

[28] N. Bloembergen, Nonlinear Optics, 4th ed. (World Scientific, Singapore, 1996).
[29] N. L. Manakov, S. I. Marmo, and A. G. Fainshtein, Zh. Eksp. Teor. Fiz. 91, 51 (1986) [Sov. Phys. JETP 64, 29 (1986)].

[30] N. L. Manakov, M. V. Frolov, A. F. Starace, and I. I. Fabrikant, J. Phys. B 33, R141 (2000), Sec. 7.

[31] N. L. Manakov, V. D. Ovsiannikov, and L. P. Rapoport, Phys. Rep. 141, 319 (1986).

[32] N. L. Manakov and A. G. Fainshtein, Teor. Mat. Fiz. 48, 375 (1981) [Theor. Math. Phys. 48, 815 (1982)].

[33] N. L. Manakov and A. G. Fainshtein, Zh. Eksp. Teor. Fiz. 79, 751 (1980) [Sov. Phys. JETP 52, 382 (1980)].

[34] N. L. Manakov, M. V. Frolov, B. Borca, and A. F. Starace, J. Phys. B 36, R49 (2003).

[35] M. L. Goldberger and K. M. Watson, Collision Theory (Wiley, New York, 1964), Chap. 8, Sec. I.

[36] N. L. Manakov, M. A. Preobrazhenskii, L. P. Rapoport, and A. G. Fainshtein, Zh. Eksp. Teor. Fiz. 75, 1243 (1978) [Sov. Phys. JETP 48, 626 (1978)].

[37] H. Sambe, Phys. Rev. A 7, 2203 (1973).

[38] P. W. Langhoff, S. T. Epstein, and M. Karplus, Rev. Mod. Phys. 44, 602 (1972).

[39] E. Mese and R. M. Potvliege, J. Phys. B 39, 431 (2006).

[40] R. M. Potvliege and R. Shakeshaft, Phys. Rev. A 40, 3061 (1989).

[41] R. M. Potvliege and R. Shakeshaft, in Atoms in Intense Laser Fields, edited by M. Gavrila (Academic, New York, 1992), p. 373.

[42] Ya. B. Zel'dovich, Zh. Eksp. Teor. Fiz. 39, 776 (1961) [Sov. Phys. JETP 12, 542 (1961)]

[43] A. I. Baz', Ya. B. Zel'dovich, and A. M. Perelomov, Scattering, Reactions and Decays in Nonrelativistic Quantum Mechanics, 2nd ed. (Nauka, Moscow, 1971).

[44] V. D. Mur, S. G. Pozdnyakov, V. S. Popov, and S. V. Popruzhenko, Pis'ma Zh. Eksp. Teor. Fiz. 75, 294 (2002) [JETP Lett. 75, 249 (2002)].

[45] V. D. Mur, S. V. Popruzhenko, V. S. Popov, and S. G. Pozdnyakov, Phys. Lett. A 316, 226 (2003).

[46] V. D. Mur, S. G. Pozdnyakov, S. V. Popruzhenko, and V. S. Popov, Phys. At. Nucl. 68, 677 (2005).

[47] N. Moiseyev, Phys. Rep. 302, 211 (1998).

[48] N. Ben-Tal, N. Moiseyev, R. Kosloff, and C. Cerjan, J. Phys. B 26, 1445 (1993).

[49] N. Moiseyev and F. Weinhold, Phys. Rev. Lett. 78, 2100 (1997).

[50] I. Gilary, P. R. Kaprálova-Žd'ánská, and N. Moiseyev, Phys. Rev. A 74, 052505 (2006).

[51] B. Borca, A. V. Flegel, M. V. Frolov, N. L. Manakov, D. B. Milošević, and A. F. Starace, Phys. Rev. Lett. 85, 732 (2000).

[52] B. Borca, D. B. Milošević, A. F. Starace, A. V. Flegel, M. V. Frolov, and N. L. Manakov, in Super-Intense Laser-Atom Physics, edited by B. Piraux and K. Rzążewsky, NATO Sci. Ser. II: Math., Phys. and Chemistry, Vol. 12 (Kluwer, Dordrecht, 2001), p. 249.

[53] A. G. Fainshtein, N. L. Manakov, and L. P. Rapoport, J. Phys. B 11, 2561 (1978).

[54] B. Borca, A. F. Starace, A. V. Flegel, M. V. Frolov, and N. L. Manakov, Phys. Rev. A 65, 051402(R) (2002).

[55] L. V. Keldysh, Zh. Eksp. Teor. Fiz. 47, 1945 (1964) [Sov. Phys. JETP 20, 1307 (1965)].

[56] V. S. Popov, Usp. Fiz. Nauk 174, 921 (2004) [Phys. Usp. 47, 855 (2004)]. 
[57] M. Yu. Kuchiev, Pis'ma Zh. Eksp. Teor. Fiz. 45, 319 (1987) [JETP Lett. 45, 404 (1987)].

[58] V. B. Berestetskii, E. M. Lifshitz, and L. P. Pitaevskii, Quantum Electrodynamics, 4th ed. (Pergamon, Oxford, 1982), Sec. 59.

[59] C. Cohen-Tannoudji, J. Dupont-Roc, and G. Grinberg, AtomPhoton Interactions (Wiley, New York, 1992), Sec. II.D.4.
[60] O. E. Alon and L. S. Cederbaum, Phys. Rev. B 68, 033105 (2003).

[61] V. N. Ostrovsky and J. B. Greenwood, J. Phys. B 38, 1867 (2005).

[62] M. V. Frolov, N. L. Manakov, E. A. Pronin, and A. F. Starace, Phys. Rev. Lett. 91, 053003 (2003). 